Pirineos, 153-154: 61 a 100, JACA; 1999

\title{
EVOLUCIÓN DE LA COMPOSICIÓN FLORÍSTICA POST-FUEGO EN UN CARRASCAL DE NAVARRA (N DE ESPAÑA)
}

\author{
R. Y. CAVERO \& A. EDERRA
}

Dpto. de Botánica. Universidad de Navarra. 31080 Pamplona. España

\begin{abstract}
RESUMEN.- Se presentan los resultados de cuatro años de estudio sobre la evolución de la composición florística de un carrascal mediterráneo sometido a fuegos controlados en Navarra ( $N$ de España). Los muestreos se han llevado a cabo en ocho cuadrados permanentes de $1 \mathrm{~m}^{2}$ de superficie, cada uno sometido a distinto tratamiento: distinto tipo de fuego, distinta situación en la parcela y adición o no de suelo no quemado. Se analizan la estrategia de regeneración de las especies que aparecen después de los fuegos y la influencia de los tratamientos en la regeneración de la flora. La evolución de la composición floristica sigue un modelo general: las especies empiezan a aparecer progresivamente, su número crece hasta obtener un máximo entre el año y los cuatro años después de los fuegos, a partir del cual disminuye. Las especies que aparecen son las mismas que habia previamente, es decir, se da un proceso de autosucesión.

ABSTRACT.- This paper presents results about floristic composition dynamics during four years after controlled fires in a mediterranean pin-oak wood in Navarra (N Spain). The samples have been taken from eight permanent $1 \mathrm{~m}^{2}$ plots subjected to different treatments: different fire type, different situation on the field, and with or without not fired soil addition. The regeneration strategies of the species and the influence of the different treatments on the flora are analysed. The floristic composition dynamics follows a general model: the species began to appear progressively. The number of species grows gradually to reach a maximum between one and three years after the fire. Later, the number of species diminishes. Post fire species are those that existed before the fire, that is to say, there is an autosuccession process.
\end{abstract}

RÉSUMÉ.- On présente les résultats de quatre ans d'étude sur l'évolution de la composition floristique d'un bois de chêne vert méditerranéen soumis à des feux contrôlés en Navarra ( $N$ de l'Espagne). Les échantillonages ont été réalisés sur huit carrés permanents d'un mètre carré de surface, chacun soumis à un traitement différent 
PIRINEOS 153-154

\begin{abstract}
selon: la sorte de feu, la difference de situation dans la parcelle et l'addition ou pas de sol non brûlé. On analyse la stratégie de régénération des espèces qui se montrent après les feux et l'influence des traitements employés sur la régénération de la flore. L'évolution de la composition floristique suit un modèle général: les espèces commencent à apparaître progressivement; leur nombre augmente jusqu'à obtenir un maximum entre une année et quatre ans après les incendies, au delà il diminue. Les espèces qui apparaîssent sont les mêmes qu'antérieurement, c'est-à-dire, qu'il y. a un processus d'autosuccession.
\end{abstract}

Keywords: Evolution, floristic composition, post-fire, pin-oak wood, Navarra, Spain.

\title{
1. Introducción
}

El fuego es un importante factor ecológico perturbador que influye en la dinámica de la vegetación de todas las regiones del mundo. Su efecto es directo sobre la estructura (AFOLAYAN, 1979; SCHAEFER, 1993; ARNO et al., 1997; FRANKLIN et al., 1997; GRAY \& FRANKLIN, 1997; LATERRA, 1997; WHITTLE et al.,1997) y composición de la vegetación (TRABAUD \& LEPART, 1980 y 1981; TRABAUD \& CHANTERAC, 1985; MAZZOLENI, 1989; AGRAVAL, 1990; CALVO et al., 1993; BOO et al., 1997; URDIROZ, 1999), así como sobre el suelo de los ecosistemas (ALMENDROS et al, 1988; CASAL et al., 1990; SCHAETZL, 1994; FERNÁNDEZ et al., 1997).

El impacto perturbador más o menos intenso del fuego depende de la temperatura que alcanza el suelo, de la frecuencia con que se da y de la estación en el que se produce. La intensidad afecta a la regeneración: a baja intensidad se favorece la regeneración de las especies rebrotadoras, a alta intensidad se favorece la recuperación de las especies germinadoras (ALLEN \& PARTRIDGE,1988; CANADELL et al., 1991; MORENO \& OECHEL, 1994) y la abundancia en el número de especies disminuye (BOO et al., 1997); también afecta al banco de semillas (ARCHIBOLD, 1979; COCKS \& STOCK, 1997; WILLIAMS \& CLARKE, 1997) y por lo tanto a la germinación de las mismas (KEELEY, 1987, 1991 y 1992; GONZÁLEZ-RABANAL \& CASAL, 1995; MARTÍNEZSÁNCHEZ et al., 1995; KEELEY \& FOTHERINGAM, 1998; KEELEY et al., 1999). Intensidades o temperaturas altas unas veces favorecen la germinación de algunas plantas como Pinus halepensis (THANOS et al., 1996; SARACINO et al., 1997), Calluna vulgaris, Erica scoparia y Cistus sp. (OJEDA et al., 1996) mientras que otras la retardan como en Arbutus unedo y Myrtus communis (MAZZOLENI, 1989; CANADELL et al., 1991). Por último, la intensidad también afecta al suelo, así a baja intensidad el suelo presenta más cantidad de materia orgánica que protege contra la erosión (TÁRREGA et al., 1996) y más nutrientes 
(SINGH, 1993; TRABAUD, 1994; CARREIRA \& NIELL, 1995) que favorecen la restauración de la vegetación (FERNÁNDEZ et al., 1997).

Otro factor a tener en cuenta en la perturbación por fuego es la frecuencia con que se produce; la alta frecuencia de incendios en una zona provoca un deterioro continuo en la vegetación tanto por la disminución del número de especies como porque alcanzan menor biomasa (COLLINS, 1989; ABRAHAMSOM \& ABRAHAMSOM, 1996). Por último, la estación del año o lo que es lo mismo, el estado en que se encuentra la vegetación cuando se produce el incendio, afecta a su regeneración; los incendios otoñales son los más favorables para la recuperación de la vegetación (AFOLAYAN, 1979; ALLEN \& PARTRIDGE, 1988; SIEG \& WRIGHT, 1996).

Tras el impacto más o menos intenso de los incendios forestales la vegetación se recupera gracias a dos mecanismos: la germinación y el rebrote (TRABAUD, 1987; PAPIÓ, 1988; KUTIEL \& KUTIEL, 1989; SCHAEFER, 1993; OJEDA et al., 1996). Muchas especies perennes sobreviven por su capacidad de rebrote (MALLIK \& GIMINGHAM, 1983); las plantas anuales y herbáceas aprovechan los cambios ambientales creados tras el fuego, como son el aumento de luminosidad (PONS, 1992), la creación de huecos vegetacionales (WILLSON, 1992), la ausencia de competencia (MALLIK \& GIMINGHAM, 1983) y el aumento de la disponibilidad de nutrientes (SINGH, 1993; TRABAUD, 1994; CARREIRA \& NIELL, 1995) para germinar y crecer más intensamente, incluso plantas que antes del incendio eran poco abundantes o estaban ausentes (TRABAUD et al., 1997).

Los ecosistemas mediterráneos han sido y son los más afectados por los incendios forestales y los más estudiados desde el punto de vista de la regeneración vegetacional; caben destacar los trabajos de SOUTO \& MONTEIRO, 1987; GÓMEZ GUTIÉRREZ et al., 1988; TÁRREGA \& LUIS, 1988a, 1988b, 1990a, 1990b; KUTIEL \& KUTIEL, 1989; MAZZOLENI, 1989; PERY, 1989; CAÑAS \& al., 1990; DE LILLIS \& TESTI, 1990; TÁRREGA et al. 1990; GARCÍA FAYOS, 1991; GRATANI \& AMADORI, 1991; LLORET \& ZEDLER, 1991; LLORET \& LÓPEZ SORIA, 1993; CABEZUDO et al., 1995; CLEMENT et al., 1996; HANLEY \& FENNER, 1997; LLORET \& VILA, 1997, etc. Las ideas más generales que se deducen de todos estos trabajos son: en primer lugar, que los ecosistemas mediterráneos tienen una gran capacidad de regeneración tras el fuego; en segundo lugar, que el restablecimiento de la comunidad original, tanto de su estructura como de su composición florística, es un proceso muy rápido, que dura unos 5 años, y sigue un modelo de autosucesión con predominio de la regeneración vegetativa; y por último, que la capacidad regenerativa de la vegetación se modifica en función de la intensidad y recurrencia del fuego.

En Navarra nunca se había estudiado la dinámica de los bosques relacionada con episodios de fuego, por lo que, dado el interés del tema, desde 1991 esta- 
mos estudiando el impacto de fuegos controlados sobre la vegetación y recuperación post-fuego en dos bosques, un carrascal mediterráneo de Quercus rotundifolia Lam. y un robledal atlántico de Quercus robur L. Así, EDERRA et al. (1997) compararon la riqueza específica encontrada en los dos bosques estudiados después de los fuegos controlados y se observa que ésta es mayor en el robledal que en el carrascal. En CAVERO \& EDERRA (1997a) se presenta para el robledal atlántico la evolución de la riqueza florística después de la perturbación, se confirma el proceso de autosucesión para este ecosistema y se observa que la recuperación es más rápida tras fuego rápido, en zonas inferiores de las parcelas quemadas y en cuadrados a los que se les ha añadido una capa delgada de suelo no quemado. CAVERO \& EDERRA (1997b) estudian la evolución de la cobertura en el carrascal mediterráneo teniendo en cuenta la influencia de tres factores, y se observa que éstos influyen en la recuperación, especialmente la intensidad del fuego, ya que el fuego lento retrasa al principio la aparición de plantas, pero después parece favorecer su permanencia, mientrás que la adición de suelo no quemado es siempre negativa para la recuperación.

En el presente trabajo damos a conocer los resultados obtenidos a lo largo de cuatro años de estudio después de fuegos controlados acerca de las formas de regeneración y de la evolución de la composición florística de un carrascal mediterráneo, teniendo en cuenta la influencia de tres factores: tipo de fuego, situación en la parcela y adición o no de suelo no quemado. Los objetivos son conocer la estrategia regenerativa de cada especie, en qué circunstancia domina una estrategia frente a otra, el comportamiento de las especies regeneradas, estudiar la evolución de la composición florística y comparar estos resultados con los obtenidos por otros autores en otros ecosistemas mediterráneos.

\section{Material y métodos}

En este trabajo queremos probar si la estrategia regenerativa de cada especie y la evolución florística están influenciadas por la intensidad del fuego, la situación en la parcela y la adición o no de suelo no quemado; y si en este carrascal se da el mismo tipo de sucesión secundaria observada por otros autores para otros ecosistemas.

\section{1 Área de estudio}

El área de estudio es un carrascal formado por Quercus rotundifolia Lam. perteneciente a la asociación Spiraeo obovatae-Quercetum rotundifoliae Rivas Goday ex Loidi \& F. Prieto 1986. Está situado en la Sierra del Perdón (zona 
Media de Navarra, España, 30TXN0830), instalado sobre sustrato básico, a una altitud entre los 750 y $785 \mathrm{~m}$, en ladera orientada al SW con una pendiente media del 30\%. Soporta un clima subhúmedo, mesotérmico, con pluviosidad anual de $600 \mathrm{~mm}$ y temperatura media anual de $13,5^{\circ} \mathrm{C}$.

\subsection{Diseño del muestreo}

En el carrascal se delimitaron tres parcelas homogéneas de 0,5 ha cada una (Figura 1). A una de ellas se le sometió a un fuego de escasa intensidad (B1) en octubre de 1991 caracterizado por una quema parcial tanto de las carrascas como del suelo, es decir, junto a árboles algo quemados quedaron otros intactos y junto a corros muy bien quemados quedaron otros con el mantillo sin quemar; a este fuego se le ha denominado fuego rápido. A otra parcela se le sometió en enero de 1992 a un fuego de intensidad moderada (B2) caracterizado por una quema total tanto de los árboles como del mantillo del suelo, quedando éste completamente desnudo; a este fuego se le ha denominado fuego lento. La tercera parcela, situada entre las dos anteriores, a la que se ha denominado «zona control», se dejó con el carrascal intacto.

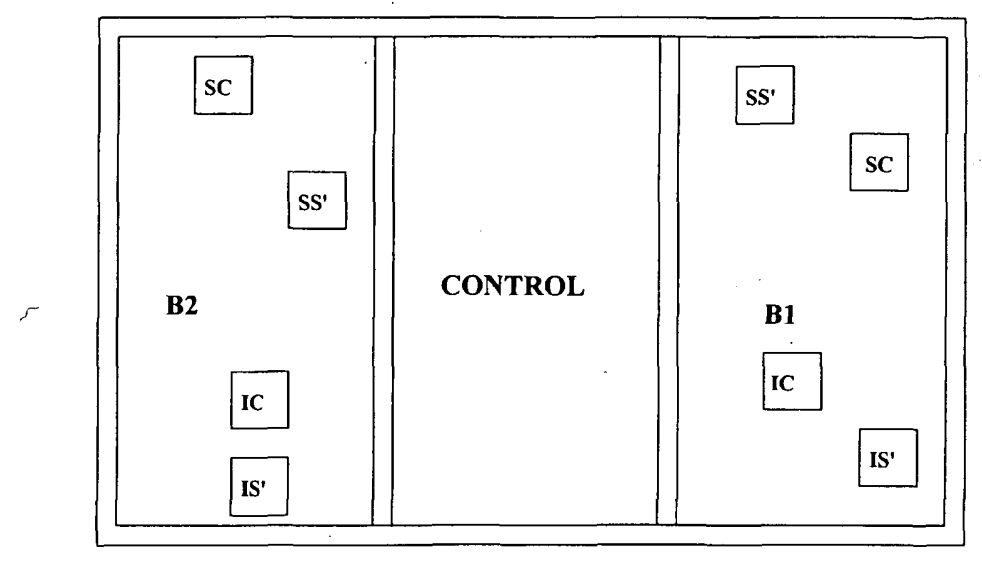

B1: cuadrado permanente situado en la parcela de fuego rápido; B2: cuadrado permanente situado en la parcela de fuego lento; I: cuadrado permanente situado en la zona inferior de las parcelas; S: cuadrado permanente situado en la zona superior de las parcelas; C: cuadrado con adición de suelo no quemado; $S^{\prime}$ : cuadrado sin adición de suelo no quemado

Figura 1. Diseño del experimento. Experimental design. 
En cada parcela quemada se instalaron cuatro cuadrados formados cada uno por cuatro placas de aluminio que delimitan $1 \mathrm{~m}^{2}$ de superficie; dos de estos cuadrados se situaron en la zona inferior de la parcela donde el suelo es más profundo y los otros dos en la zona superior de la misma; en esta zona el suelo es pedregoso, la escorrentia muy fuerte y durante la mayor parte del año existen vientos persistentes y desecantes. A uno de los dos cuadrados de cada zona se le adicionó $2-3 \mathrm{~cm}$ del horizonte superior de suelo forestal no quemado procedente de los alrededores. En total, se han instalado ocho cuadrados permanentes cada uno con características diferenciadas: distinto tipo de fuego, distinta situación en la parcela y adición o no de suelo no quemado. Por tanto, en el diseño experimental empleado no hay replicación de los tratamientos sino pseudoreplicaciones (HURLBERT, 1984), por lo que los resultados que obtengamos mostrarán, más que pruebas, tendencias en la regeneración. En la Tabla 1 se indican esquematizados los tratamientos y sus características.

\begin{tabular}{|l|l|l|}
\hline \multicolumn{2}{|c|}{ Tratamiento } & \multicolumn{1}{|c|}{ Características } \\
\hline Fuego & Rápido (B1) & $\begin{array}{l}\text { De escasa intensidad. Quema de } \\
\text { carrascas y de mantillo en mosaico: } \\
\text { Zonas bien quemadas junto a zonas } \\
\text { poco quemadas. }\end{array}$ \\
\hline Situación en la parcela & Zento (B2) & $\begin{array}{l}\text { De intensidad moderada. Quema } \\
\text { total de la vegetación. Suelo desnudo }\end{array}$ \\
\hline Adición o no de suelo & Con adición (C) & $\begin{array}{l}\text { Suelo profundo. Poca escorrentía } \\
\text { Mínimo efecto del viento }\end{array}$ \\
\hline & Sin adición (S') & $\begin{array}{l}\text { 2-3 cm del horizonte superior de suelo } \\
\text { no quemado de los alrededores }\end{array}$ \\
\hline $\begin{array}{l}\text { Suelo tal como queda después de los } \\
\text { fuegos }\end{array}$ \\
\hline
\end{tabular}

Tabla 1. Resumen de los tratamientos empleados. Summary of treatments.

\subsection{Realización de los muestreos}

Antes de poner en práctica los fuegos controlados se procedió al estudio de la flora vascular del carrascal; después de los fuegos y durante cuatro años 
se han realizado muestreos periódicos para la recogida de datos de presenciaausencia y formas de regeneración de las especies en los ocho cuadrados permanentes y poder conocer las tendencias de la composición florística, de la riqueza específica y de su evolución a lo largo del tiempo, así como las formas de regeneración de las especies. Se han anotado por separado los datos de cada uno de los cuadrados, en ausencia de replicaciones verdaderas (HURLBERT, 1984), para observar tendencias de la influencia de los tres tratamientos en todos estos aspectos de la regeneración post-fuego. La zona control ha servido tanto para completar la lista de especies presentes en el carrascal como para la realización de otros estudios distintos del presente.

Los muestreos en los cuadrados permanentes, numerados correlativamente, se realizaron en las fechas siguientes:

- Año 1992: $1=10$ de marzo; $2=9$ de abril; $3=5$ de mayo; $4=11$ de junio; $5=14$ de julio; $6=20$ de agosto; $7=22$ de septiembre.

- Año 1993: 8=22 de febrero; $9=7$ de abril; $10=26$ de abril; $11=31$ de mayo; $12=5$ de julio; $13=27$ de julio; $14=7$ de septiembre; $15=6$ de octubre; $16=3$ de noviembre; $17=13$ de diciembre.

- Año 1994: $18=3$ de marzo; $19=24$ de marzo; $20=28$ de abril; $21=24$ de junio; $22=27$ de julio; $23=28$ de septiembre; $24=3$ de noviembre.

- Año 1995: 25 =15 de marzo; $26=4$ de julio; $27=9$ de octubre.

Para la nomenclatura científica de las especies y los nombres de los autores se ha seguido siempre a TUTIN et al. (1964-1980).

\subsection{Estrategias de regeneración}

Mediante el estudio de los tipos biológicos y la observación detenida de los fenotipos de los taxa que han ido apareciendo en los cuadrados permanentes, principalmente durante los primeros muestreos, hemos podido diferenciar en las especies recolonizadoras ocho tipos de estrategia de regeneración (Tabla 2) según sea su principal vía de establecimiento en el terreno después de la perturbación, como en este caso han sido los fuegos. Las ocho estrategias pueden reagruparse en tres tendencias principales: a) especies rebrotadoras, que son hemicriptófitos, geófitos, caméfitos y fanerófitos, que sólo se regeneran vegetativamente; b) especies germinadoras, que son especies anuales y bianuales o vivaces que se reinstalan mediante germinación, pues las plantas que han sufrido la alteración son incapaces de retoñar, y c) especies rebrotadoras-germinadoras, que son las que utilizan las dos formas de regeneración, de manera que durante los primeros meses después de la 
PIRINEOS 153-154

\begin{tabular}{|c|c|c|}
\hline Estrategia & $\begin{array}{l}\text { Identificador } \\
\text { de estrategia }\end{array}$ & Descripción \\
\hline \multirow[t]{2}{*}{ a, Rebrotadoras } & 1 & $\begin{array}{l}\text { Plantas rebrotadoras mediante } \\
\text { órganos superficiales }\end{array}$ \\
\hline & 2 & $\begin{array}{l}\text { Plantas rebrotadoras mediante } \\
\text { órganos subterráneos }\end{array}$ \\
\hline \multirow[t]{2}{*}{ b, Germinadoras } & 3 & Plantas germinadoras oportunistas \\
\hline & 4 & Plantas germinadoras no oportunistas \\
\hline \multirow{4}{*}{$\begin{array}{l}\text { c, Rebrotadoras- } \\
\text { germinadoras }\end{array}$} & 5 & $\begin{array}{l}\text { Plantas rebrotadoras mediante } \\
\text { órganos superficiales y germinadoras }\end{array}$ \\
\hline & 6 & $\begin{array}{l}\text { Plantas rebrotadoras mediante } \\
\text { órganos subterráneos y germinadoras }\end{array}$ \\
\hline & 7 & $\begin{array}{l}\text { Plantas rebrotadoras mediante } \\
\text { órganos superficiales y germinadoras, } \\
\text { preferentemente germinadoras }\end{array}$ \\
\hline & 8 & $\begin{array}{l}\text { Plantas rebrotadoras mediante } \\
\text { órganos superficiales y germinadoras, } \\
\text { preferentemente rebrotadoras }\end{array}$ \\
\hline
\end{tabular}

Tabla 2. Distintas estrategias de regeneración de las especies recolonizadoras. Regeneration strategies of the recolonising species.

perturbación domina la regeneración por rebrote, y posteriormente, la regeneración por semilla o vicecersa.

\section{Resultados y discusión}

Los resultados obtenidos aparecen en anexo en forma de tablas. En la Tabla 3 se indica la evolución de la composición florística a lo largo del tiempo; en la Tabla 4 figuran todos los taxa que han aparecido a lo largo de los cuatro años de estudio, tanto en las parcelas quemadas como en la zona control sin quemar o en toda el área antes de provocar los fuegos.

\subsection{Autosucesión}

Sin considerar los taxa no identificados a nivel al menos genérico, puesto que de ellos no podemos saber si ya estaban antes de los fuegos o no, son 68 los taxa que han aparecido a lo largo del tiempo en los cuadrados permanentes, de los que una mayoría, 56, ya estaban en el bosque antes de los fuegos. 
Sin embargo, su comportamiento temporal ha sido diferente: 42 de los 68 están presentes en el último año de estudio (Tabla 4 , año número 4 en negrita), de lo cual podría deducirse que representan más o menos cómo es o será la flora del lugar si continúa el proceso de recuperación tras los fuegos.

Por otra parte, de los 42 taxa que reconocimos en el cuarto año de seguimiento de la recuperación, 28 (66,6\%, todo en negrita en la Tabla 4) se han mantenido con continuidad en todos los cuadrados en que aparecieron; estos serían por tanto los taxa mejor adaptados al terreno. Cabe destacar que, de estos 28 , los más constantes a lo largo de los cuatro años, los más frecuentes y los que más pronto aparecieron son precisamente los característicos del carrascal, de sus orlas arbustivas o de las comunidades de su serie de vegetación, por ejemplo Quercus rotundifolia, Brachypodium retusum, Genista hispanica ssp. occidentalis, Cistus salvifolius, Rubia peregrina, Teucrium chamaedrys, Anthyllis vulneraria, Thymus vulgaris y Erica vagans. Otras especies, como Genista scorpius y Thymelaea ruizii, aunque no aparecen más que en 2 cuadrados permanentes, también han sido constantes en los cuatro años.

De todo esto deducimos que en este carrascal se da el proceso de autosucesión de la vegetación reseñado por otros autores para otros ecosistemas (TRABAUD, 1985, 1990, 1991a y 1991b; TRABAUD \& LEPART, 1980 y 1981; PRODON et al., 1984; KUTIEL \& KUTIEL, 1989; TÁRREGA \& LUIS, 1990a y 1990b; DE LILLIS \& TESTI, 1990; CASAL et al., 1990 y CAÑAS et al., 1990; CLEMENTE et al., 1996). Interpretamos que el proceso de autosucesión se da a pesar de la gran cantidad de plantas que no se han vuelto a incorporar a la flora del lugar (Tabla 4: 58 especies fueron recolectadas sólo antes de los fuegos) o que no han sobrevivido hasta el cuarto año de estudio (Tabla 4: 26 de 68 más 12 no identificadas a nivel genérico).

Después de la perturbación predomina la regeneración vegetativa de las especies perennes; a partir del segundo y tercer muestreo en los cuadrados de fuego lento - excepto en B2SS' - y del cuarto muestreo en los de fuego rápido - excepto $\mathrm{B} \mathrm{SS}^{\prime}$ - empiezan a aparecer las especies terófitas (Tabla 3). El predominio de la estrategia rebrotadora en los primeros momentos después del fuego es un hecho muy frecuente en los ecosistemas mediterráneos (CASAL et. al, 1990; TÁRREGA \& LUIS, 1988a, 1988b y 1990b; TRABAUD, 1991a y 1991b) y responde a un mecanismo de adaptación de éstos frente al fuego; de hecho, podemos decir que todas las especies típicas del carrascal son preferentemente o estrictamente rebrotadoras (estrategias 1, 5, 6,7 y 8 , tablas 3 y 4 ), mientras que las que germinan, aunque también pueden llegar a ser muy abundantes en los carrascales, son sobre todo características de matorrales o pastizales de la serie de vegetación. Nuestros resultados en este sentido concuerdan con los obtenidos por PAPIÓ (1988) en Garraf (Cataluña). 
En cuanto a la posible influencia de la intensidad del fuego en la restauración de las plantas, encontramos que 31 taxa aparecen sólo en cuadrados del área quemada con fuego lento (B2), 16 taxa aparecen sólo en cuadrados del área quemada con fuego rápido (B1), y 33 en cuadrados de ambos tipos de fuego, es decir, fuego rápido y fuego lento. Apreciamos que después del fuego lento, que en principio se supone más destructivo, la regeneración del carrascal es muy favorable, e incluso, considerando el número de taxa diferentes que aparecen, mejor que después del fuego rápido, ya que en total han aparecido 64 taxa en cuadrados B2 frente a 47 en cuadrados B1. Esta diferencia a favor de la recuperación de especies en cuadrados de fuego lento se debe en parte a dos hechos destacables, que también han sido mencionados por otros autores (KRUGER, 1983 y PAPIÓ, 1988). El primero, la gran cantidad de plantas oportunistas que aparecen (resaltadas sobre fondo oscuro en la Tabla 4, estrategia 3 ) en los cuadrados B2, 10, frente a los B1, sólo 4 . El segundo, el número también elevado de plantas rebrotadoras con órganos subterráneos, tipo bulbo y rizoma (Tablas 3 y 4, estrategia 2); de hecho, todas las plantas de este tipo, 13, han aparecido en los cuadrados B2, frente a 8 que lo han hecho en los B1. Ambos hechos pueden explicarse porque después del fuego lento el suelo queda completamente desnudo, de forma que la disminución de la cobertura vegetal facilita el desarrollo de los geófitos y la implantación de plantas foráneas, si bien el número de estas especies va disminuyendo al restablecerse la vegetación del carrascal.

Por otra parté, tanto en los cuadrados B1 como en los B2 resulta llamativa la presencia de cotiledones y Poáceas que no hemos logrado determinar debido a su fugacidad (Tabla 4, 8 taxa en 8 cuadrados B1 y 6 taxa en 8 cuadrados B2). La mayoría de estos taxa aparecieron en cuadrados situados en las zonas superiores de las parcelas (cuadrados S), donde el suelo es bastante pedregoso y la escorrentía más fuerte que en los inferiores, circunstancias que dificultan la regeneración de la vegetación, por lo que todos estos taxa inconcretos han desaparecido en poco tiempo, sin conseguir desarrollarse hasta ejemplares adultos identificables a nivel de especie.

\subsection{Estrategias de regeneración}

A partir de los datos de las tablas 3 y 4 , se obtiene:

- 30 taxa utilizan la estrategia rebrotadora. De ellos, 17 rebrotan mediante órganos aéreos, es decir, son hemicriptófitos, caméfitos o fanerófitos (estrategia 1, Tabla 5) y 13 rebrotan mediante órganos subterráneos, es decir, son geófitos (estrategia 2, Tabla 6)

- 26 taxa, sin considerar aquellos no identificados a nivel al menos genérico, son germinadores (Tabla 7). De ellos consideramos que 12 son ger- 
minadores oportunistas (estrategia 3, sobre fondo oscuro) y 14 germinadores no oportunistas (estrategia 4 )

- 12 taxa son rebrotadores-germinadores (estrategias 5, 6, 7 y 8, Tabla 8).

Cabe destacar que la estrategia de regeneración de cada especie a nivel individual es la misma en todas las distintas situaciones estudiadas, es decir, los tratamientos no influyen en la estrategia de regeneración de cada especie, pero sí lo hacen, como veremos más adelante, en el conjunto de la flora.

La mayoría de nuestras observaciones concuerdan con las de PAPIÓ (1988), salvo en los casos de Aphyllantes monspeliensis y Avenula bromoides, que este autor incluye en el grupo de rebrotadoras, mientras que nosotros hemos encontrado suficientes plántulas de ambas como para incluirlas, la primera en el grupo de rebrotadoras-germinadoras, y la segunda en el grupo de germinadoras. Estas discrepancias podrían explicarse, en principio, por las distintas intensidades de las perturbaciones. Pero, si profundizamos en este aspecto, vemos que el incendio de Garraf (PAPIÓ, 1988) tuvo una intensidad entre moderada y fuerte, por lo que, según la bibliografía (ALLEN \& PARTRIDGE,1988; CANADELL et al., 1991; MORENO \& OECHEL, 1994) debería dominar la estrategia germinadora frente a la rebrotadora, cosa que en Aphyllantes monspeliensis y Avenula bromoides no ocurre. Por otra parte, nuestros fuegos fueron de intensidades menores (escasa y moderada), por lo que debería dominar la estrategia rebrotadora frente a la germinadora. Aphyllantes monspeliensis sí rebrota, aunque también germina, pero Avenula bromoides se comporta como germinadora. Por tanto, creemos que, para estas dos especies, la intensidad de las perturbaciones no explica las diferencias observadas en su estrategia de regeneración, tanto respecto a PAPIÓ (1988) como a la bibliografía mencionada más arriba. Debe haber otros factores, como pueden ser diferente tipo de suelo, de clima, de pendiente, de iluminación, etc., que podrían explicar las discrepancias observadas.

\subsubsection{Comportamiento de las especies rebrotadoras}

Algunas de las plantas rebrotadoras por órganos aéreos, junto con las geófitas y varias rebrotadoras-germinadoras aparecen ya en los primeros muestreos (Tablas 3 y 5). De aquí se deduce la gran importancia de estas plantas en la dinámica de la recuperación tras-fuego. Cabe destacar entre éstas, por su buena representación en los cuadrados permanentes y por su importancia como especies características del carrascal Festuca gr. rubra, Quercus rotundifolia y Teucrium chamaedrys.

Respecto a las geófitas (estrategia 2), es llamativo su ciclo de vida, que parece diferente en los cuadrados situados en la parcela de fuego rápido y los 
PIRINEOS 153-154

\begin{tabular}{|c|c|c|}
\hline $\begin{array}{l}\text { Rebrotadores } \\
\text { por órganos aéreos }\end{array}$ & Cuadrados & $\begin{array}{l}\text { Años correlativos de aparición } \\
\text { en cada cuadrado }\end{array}$ \\
\hline Bromus erectus & B1IC & 1 \\
\hline Carex hallerana & B1SS' $^{\prime}$ & 1234 \\
\hline Dactylis glomerata & B1IC & 34 \\
\hline Dianthus hispanicus & B2SC & 234 \\
\hline Euphorbia minuta & B2IS' $^{\prime}$ & 1234 \\
\hline Festuca grupo rubra & $\begin{array}{l}\text { B1IC, B1IS', B1SC, B1SS' } \\
\text { B2IC, B2IS', B2SS' }\end{array}$ & $\begin{array}{l}23,1,14,134 \\
1,1,1 \\
\end{array}$ \\
\hline Galium fruticescens & B2IC & 1234 \\
\hline Galium sp. & B2IS', B2SS' & 1,1234 \\
\hline Phlomis lychnitis & B2IC & 12 \\
\hline Polygala vulgaris & B2IS' $^{\prime}$ & 23 \\
\hline Potentilla tabernaemontani & $\begin{array}{l}\text { B1SS', } \\
\text { B2SS' }^{\prime}\end{array}$ & \begin{tabular}{|l|}
1234 \\
2 \\
\end{tabular} \\
\hline Quercus faginea & B2IS' $^{\prime}$ & 1234 \\
\hline Quercus rotundifolia & $\begin{array}{l}\text { B1IC, B1IS', B1SC, B1SS', } \\
\text { B2IC, B2IS', B2SC, B2SS' }\end{array}$ & \begin{tabular}{|l|}
$1234,1234,1234,1234$ \\
$1234,1234,1234,1234$ \\
\end{tabular} \\
\hline Rhamnus saxatilis & B1SS $^{\prime}$ & 1 \\
\hline Scorzonera graminifolia & $\begin{array}{l}\text { B1IC, } \\
\text { B2IS', B2SC } \\
\end{array}$ & \begin{tabular}{|l|}
3 \\
1,12 \\
\end{tabular} \\
\hline Teucrium chamaedrys & $\begin{array}{l}\text { B1IC, } \\
\text { B2IS', B2SC, B2SS' }\end{array}$ & $\begin{array}{l}1234 \\
1234,4,1234 \\
\end{array}$ \\
\hline Thymelaea ruizii & $\begin{array}{l}\text { B1SC, } \\
\text { B2IS' }\end{array}$ & \begin{tabular}{|l|}
234 \\
1234
\end{tabular} \\
\hline
\end{tabular}

Tabla 5. Especies rebrotadoras mediante órganos aéreos: Cuadrados y años de aparición en cada cuadrado. En negrita las especies presentes en el último año de muestreo y los cuadrados en que permanecen.

Resprouting species by aerial organs. Plots and years of appearance in each plot. In bold characters species present in the last year and permanent plots where they remain.

de la parcela de fuego lento (Tablas 3,4 y 6). En fuego rápido (B1), el ciclo es más o menos estacional durante los cuatro años de muestreo, excepto en los casos de Fritillaria pyrenaica en B1SS', Narcissus assoanus en B1IS'y Crocus nudiflorus en B1SC, que aparecen y desaparecen durante el primer año después de la perturbación. Por el contrario, en fuego lento (B2) desaparecen o no brotan muchas plantas geófitas; así, Narcissus assoanus brota el primer y segundo año después de la perturbación, Anthericum liliago, el primer año, ambos en B2IC; Muscari neglectum, Crocus nudiflorus, Narcissus assoanus, Thalictrum tuberosum y Allium sphaerocephalum aparecen sólo durante el segundo año en B2IC, B2IS'y B2SC. Así pues, como era de esperar, el fuego lento perturba en mayor 
grado a los geófitos que el fuego rápido, pues, si bien todas las geófitas han conseguido brotar en algún momento en algún cuadrado B2, su permanencia es menor y su brotación mucho más irregular que en los B1.

Otro hecho destacable es que en el último año de muestreo, de todas las geófitas, sólo permanecen las que tienen rizomas o tubérculos radicales, mientras que ninguna de las bulbosas ha brotado. Pudimos observar que en el segundo y tercer año de muestreo muchos bulbos, sobre todo en el área quemada con fuego lento, habían desarrollado raíces contráctiles. La desaparición de la cobertura y el consiguiente aumento de iluminación pueden estimular el rebrote de los bulbos (KRUGER, 1983), pero parece que también pueden provocar la aparición de dicho tipo de raíces, que sirven para ajustar

\begin{tabular}{|c|c|c|}
\hline Rebrotadores geófitos & Cuadrados & $\begin{array}{l}\text { Años de aparición correlativos } \\
\text { en cada cuadrado }\end{array}$ \\
\hline Allium sphaerocephalum & B2SC & 2 \\
\hline Anthericum liliago & B2IC & 1 \\
\hline Brachypodium phoenicoides & B2SS' & 12 \\
\hline Brachypodium retusum & $\begin{array}{l}\text { B1IC, B1IS', B1SC, B1SS', } \\
\text { B2IC, B2IS', B2SC, B2SS' }^{\prime}\end{array}$ & \begin{tabular}{|l|}
$1234,1234,1234,1234$, \\
$1234,1234,1234,1234$,
\end{tabular} \\
\hline Conopodium majus & $\begin{array}{l}\text { B1SS', } \\
\text { B2SS' }^{\prime}\end{array}$ & $\begin{array}{l}123 \\
\mathbf{1 2 3 4} \\
\end{array}$ \\
\hline Crocus nevadensis & B2SC & 23 \\
\hline Crocus nudiflorus & $\begin{array}{l}\text { B1IS', B1SC, B1SS', } \\
\text { B2IC, B2IS', B2SC }\end{array}$ & $\begin{array}{l}123,1,1 \\
2,2,2\end{array}$ \\
\hline Filipendula vulgaris & $\begin{array}{l}\text { B1IC, B1SS', } \\
\text { B2SS' }^{\prime}\end{array}$ & \begin{tabular}{|l|}
1234,13 \\
1234 \\
\end{tabular} \\
\hline Fritillaria pyrenaica & $\begin{array}{l}\text { B1SS', } \\
\text { B2SS' }\end{array}$ & $\begin{array}{l}1, \\
2 \\
\end{array}$ \\
\hline Muscari neglectum & $\mathrm{B} 2 \mathrm{IC}, \mathrm{B} 2 \mathrm{SC}$ & 2,2 \\
\hline Narcissus assoanus & $\begin{array}{l}\text { B1IS', }^{\prime} \\
\text { B2IC, B2SC, B2SS' }\end{array}$ & \begin{tabular}{|l|}
1 \\
$12,2,123$ \\
\end{tabular} \\
\hline Rubia peregrina & $\begin{array}{l}\text { B1IC, B1IS', B1SC, B1SS', } \\
\text { B2IS' }^{\prime}\end{array}$ & $\begin{array}{l}1234,1234,1234,1234, \\
1234\end{array}$ \\
\hline Thalictrum tuberosum & $\begin{array}{l}\text { B1IC, B1IS', } \\
\text { B2IC, B2SC, B2SS }\end{array}$ & \begin{tabular}{|l|}
1234,1234 \\
$123,2,1234$
\end{tabular} \\
\hline
\end{tabular}

Tabla 6. Especies rebrotadoras mediante órganos subterráneos. Cuadrados y años en que aparecen en cada cuadrado. En negrita las especies presentes en el último año de muestreo y los cuadrados en que permanecen.

Resprouting species by subterranean organs. Plots and years of appearance in each plot. In bold characters species present in the last year and permanent plots where they remain. 
la profundidad a la que se situan los bulbos; así, este hecho, junto con el aumento progresivo de cobertura después del segundo año, pueden ser la causa de que los bulbos queden a mayor profundidad y sin florecer, en estado de semilatencia, a partir del tercer y cuarto año post-fuego.

\subsubsection{Comportamiento de las especies germinadoras}

En las Tablas 3, 4 y 7 se observa que de las 26 plantas germinadoras, 12 aparecen en los cuadrados de la parcela de fuego lento (B2), 5 en los de la parcela de fuego rápido (B1) y 9 en los de ambos tipos de fuego (B1 y B2); es en el fuego lento, donde las condiciones son más favorables -suelo desnudo y rico en nutrientes proporcionados por las cenizas- donde más plantas germinadoras se restablecen.

Entre las plantas germinadoras (Tabla 7) hemos realizado dos grupos: 3 y 4. El 3, sobre fondo oscuro en Tablas 4 y 7 , corresponde a plantas que no estaban en el carrascal antes de la perturbación y aprovechan la nueva situación creada para instalarse. Son las especies que llamamos «oportunistas», que deben proceder de los alrededores, aunque quizá también estuvieran en estado latente como semillas en el banco del suelo (THOMPSON, 1992), pero sin poder germinar por no encontrar en el carrascal intacto las condiciones que necesitan (PICKETT \& WHITE, 1985; PICKETT et al., 1989; POSSINGHAM et al., 1995). El grupo 4 incluye especies propias de claros de carrascal y de sus matorrales subseriales, donde son muy frecuentes, pero que ya estaban también presentes en el bosque antes de los fuegos. A estas plantas les denominamos «germinadoras no oportunistas» o, simplemente, «no oportunistas».

A. Las plantas germinadoras-oportunistas son 12, de las que 8 aparecen sólo en los cuadrados de la parcela de fuego lento (B2), 2, sólo en los de la parcela de fuego rápido (B1) y otras 2, en los de ambas parcelas (B1 y B2). De estos números se deduce que es en los cuadrados de fuego lento donde más número de plantas oportunistas hay, seguramente porque encuentran las condiciones más favorables para su establecimiento (WILLSON, 1992).

De las 12 plantas oportunistas, 6 están sólo en los cuadrados inferiores (I): Taraxacum sp., Picris echioides, P. hieracioides, Potentilla reptans, Vicia-Lathyrus y Sonchus asper; otras 3 solo en los superiores (S): Cerastium semidecandrum, Galium aparine y Hordeum sp.; y por último, otras 3 aparecen tanto en los cuadrados I como en los S: Cirsium arvense, Cirsium vulgare y Sonchus oleraceus. Por tanto, se aprecia que la mayoría de las especies que muestran cierta selectividad para los cuadrados aparecen en las zonas inferiores de las parcelas donde el suelo es más profundo y por lo tanto la nascencia y desarrollo más fácil (como ya hemos dicho anteriormente las partes altas de las parcelas son muy pedregosas y con mucha escorrentía). 


\begin{tabular}{|c|c|c|}
\hline Germinadoras & Cuadrados & $\begin{array}{l}\text { Años correlativos de aparición } \\
\text { en cada cuadrado }\end{array}$ \\
\hline Aira caryophyllea & B2SC & 4 \\
\hline Anthyllis vulneraria & $\begin{array}{l}\text { B1SC, B1SS', } \\
\text { B2SC }\end{array}$ & $\begin{array}{l}34,124, \\
1234\end{array}$ \\
\hline Argyrolobium zanonii & $\begin{array}{l}\mathrm{B} 1 \mathrm{IS}^{\prime}, \mathrm{B} 1 \mathrm{SC}, \\
\mathrm{B} 2 \mathrm{IC}, \mathrm{B} 2 \mathrm{SC}\end{array}$ & $\begin{array}{l}12,234 \\
1234,1234\end{array}$ \\
\hline Asterolinum linum-stellatum & $\begin{array}{l}\text { B1SC, B1SS', } \\
\text { B2IC, B2SC }\end{array}$ & $\begin{array}{l}1234,1234 \\
2,234\end{array}$ \\
\hline Avenula bromoides & $\begin{array}{l}\text { B1IC, } \\
\text { B2SC }\end{array}$ & $\begin{array}{l}234 \\
234\end{array}$ \\
\hline Blackstonia perfoliata & ${\mathrm{B} 1 S S^{\prime}}^{\prime}$ & 3 \\
\hline Bombycilaena erecta & $\mathrm{B} 2 \mathrm{SC}$ & 3 \\
\hline Cerastium semidecandrum & B2SC & 3 \\
\hline Cirsium arvense & B1IS', B1SC, B1SS' & $123,2,34$ \\
\hline Cirsium vulgare & $\begin{array}{l}\text { B1IS', B1SS', } \\
\text { B2IS', B2SS' }\end{array}$ & $\begin{array}{l}123,1234 \\
1234,12\end{array}$ \\
\hline Coronilla minima & $\mathrm{B} 2 \mathrm{IS}^{\prime}$ & 12 \\
\hline Crucianella angustifolia & B1SC & 34 \\
\hline Eryngium campestre & B1IC & 24 \\
\hline Euphorbia exigua & $\begin{array}{l}\text { B1SS', } \\
\text { B2SC }\end{array}$ & $\begin{array}{ll}34 \\
234 \\
\end{array}$ \\
\hline Fumana ericoides & B2SC & 1234 \\
\hline Galium aparine. & $\mathrm{B} 2 \mathrm{SS}^{\prime}$ & 3 \\
\hline Gastridium ventricosum & $\begin{array}{l}\text { B1SC, } \\
\text { B2SC }\end{array}$ & $\begin{array}{l}234 \\
234 \\
\end{array}$ \\
\hline Hordeum sp. & B2SS & 2 \\
\hline Ononis minutissima & $\begin{array}{l}\text { B1SS', } \\
\text { B2SC }\end{array}$ & $\begin{array}{l}1, \\
1234\end{array}$ \\
\hline Picris echioides & B2IC & 1. \\
\hline Picris hieracioides. & $\mathrm{B} 2 \mathrm{IC}$ & 2.2 \\
\hline Potentilla reptans & B2IS & 234 \\
\hline Sonchus asper & B2IC & 1 \\
\hline Sonchus oleraceus & $\begin{array}{l}\text { B1IC, B1SS', } \\
\text { B2IS', B2SS' }\end{array}$ & $\begin{array}{l}3,12 \\
1,1\end{array}$ \\
\hline Taraxacum sp. & B1IC & 2.2 \\
\hline Vicia-Lathyrus & B2IS' & 24 \\
\hline
\end{tabular}

Tabla 7. Especies germinadoras. Cuadrados y años en que aparecen en cada cuadrado. Sobre fondo oscuro las germinadoras-oportunistas (estrategia 3); en fondo claro las demás germinadoras, no oportunistas (estrategia 4). En negrita las especies presentes en el último año de muestreo y los cuadrados en que permanecen.

Germinating species. Plots and years of appearance in each plot. On a dark background the germinatingopportunist plants (strategie 3); on a light background the rest of the germinating plants, not opportunist (strategie 4). In bold characters species present in the last year and permanent plots where they remain. 
En cuanto a la influencia de la adición de suelo no quemado en el establecimiento de plantas oportunistas, se observa que aparecen 5 plantas en los cuadrados con adición de suelo no quemado (C): Taraxacum sp., Cerastium semidecandrum, Picris echioides, Picris hieracioides y Sonchus asper; otras 5 en los cuadrados sin adición de suelo (S'): Cirsium vulgare, Galium aparine, Hordeum sp., Potentilla reptans y Vicia-Lathyrus; y 2, aparecen tanto en unos como en otros: Cirsium arvense y Sonchus oleraceus. Parece por tanto que este tratamiento no afecta en la presencia o ausencia de plantas oportunistas.

Un dato llamativo de todo este estudio es la gran amplitud de hábitats que es capaz de colonizar Sonchus oleraceus: es la única especie encontrada capaz de vivir en fuego rápido y lento, en la parte superior de la parcela y en la parte inferior, y en los cuadrados con adición de suelo y en los cuadrados sin adición. También Cirsium arvense aparece en varios cuadrados, pero siempre sin adición de suelo no quemado.

Por último, cabe preguntarse cuándo aparecen y desaparecen estas plantas, es decir, cúal es su ciclo de vida. En este sentido, (Tablas 3 y 7 ) se observa que: a/ algunas plantas aparecen y desaparecen durante el primer año, segundo año y tercer año, así durante el primer año aparecen y desaparecen Picris echioides y Sonchus asper, durante el segundo año, Hordeum sp., y Picris hieracioides; y durante el tercer año, Taraxacum sp., Cerastium semidecandrum y Galium aparine; b/ otras aparecen casi siempre, es decir, durante todos los años de estudio hasta el final, Cirsium arvense y Cirsium vulgare, o bien desde el segundo año hasta el final, Potentilla reptans y Vicia-Lathyrus; y c/ Sonchus oleraceus aparece según los cuadrados, durante los distintos años de muestreo, pero en ningún caso llega hasta el último año. Por tanto, sólo 4 plantaș oportunistas logran permanecer hasta el final del estudio, que, considerandolas en relación a nuestros resultados globales, son en realidad sólo 4 de las 68 plantas que han aparecido en los cuadrados, 4 de las 42 plantas las que logran establecerse en el último año de estudio, lo que nos lleva a pensar que la regeneración del carrascal está bastante avanzada puesto que desaparecen las condiciones favorables para la persistencia de estas plantas oportunistas.

B. El resto de las plantas germinadoras encontradas en los cuadrados a lo largo del tiempo (Tabla 7) corresponden a especies frecuentes en claros de carrascal o en los matorrales de degradación, especies no oportunistas, y son 14. De ellas, 4 aparecen sólo en los cuadrados de la parcela de fuego lento (B2), 3 sólo en los de la parcela de fuego rápido (B1) y 7 aparecen tanto en los cuadrados situados en la parcela de fuego lento como en los de fuego rápido (B2 y B1). Es decir, la mitad de las especies germinadoras no oportunistas encontradas en los cuadrados aparecen tanto en los cuadrados de fuego rápido como en los de lento, ya que en ambas parcelas se crean condiciones adecuadas para su germinación (formación de claros en el bosque donde luz y 
nutrientes son abundantes para la regeneración). Este hecho difiere del encontrado para las plantas oportunistas que, como hemos dicho anteriormente, son más abundantes en los cuadrados de la parcela de fuego lento, donde la liberación de nutrientes y el aumento de la iluminación es aún mayor (véase Tabla 1).

De las especies germinadoras no oportunistas, 9 aparecen sólo en los cuadrados superiores (S): Aira caryophyllea, Anthyllis vulneraria, Blackstonia perfoliata, Bombycilaena erecta, Crucianella angustifolia, Euphorbia exigua, Fumana ericoides, Gastridium ventricosum y Ononis minutissima; 2, en los inferiores: Coronilla minima y Eryngium campestre; y, por último, otras 3 aparecen tanto en los cuadrados inferiores como en los superiores (I y S): Argyrolobium zanonii, Asterolinum linum-stellatum y Avenula bromoides. El comportamiento de estas plantas vuelve a ser diferente al de las plantas oportunistas, que eran más frecuentes en los cuadrados inferiores. Quizás en las partes superiores de las parcelas, más pedregosas, se dan condiciones más parecidas a las de los matorrales y pastos de sustitución del carrascal, o bien llegan más semillas autóctonas del carrascal que están más adaptadas a estas circunstancias. Además cabe mencionar que estas partes superiores son más venteadas, lo cual quizás también favorezca la llegada de semillas (WILLSON, 1992).

También es interesante analizar la influencia de la adición o no de suelo no quemado en estas plantas. Encontramos que: 7 de las 14 están sólo en los cuadrados con adición de suelo no quemado (C): Aira caryophyllea, Avenula bromoides, Bombycilaena erecta, Crucianella angustifolia, Eryngium campestre, Fumana ericoides y Gastridium ventricosum; 2 , en los cuadrados sin adición ( $\left.S^{\prime}\right)$ : Blackstonia perfoliata y Coronilla minima; y 5 en ambos tipos de cuadrados (C y S'): Anthyllis vulneraria, Aroyrolobium zanonii, Asterolinum linum-stellatum, Euphorbia exigua y Ononis minutissima. Así pues, en los cuadrados con adición de suelo no quemado germinan más especies de plantas no oportunistas que en los cuadrados sin adición, seguramente debido al aporte extra de semillas de estas plantas al añadir el suelo no quemado. Nuevamente se observan diferencias entre el comportamiento de las plantas oportunistas y las no oportunistas en relación a este tratamiento.

Y por último, en cuanto al ciclo de vida de estas plantas no oportunistas, es decir, cúando germinan y si logran o no establecerse durante los 4 años de nuestra experiencia, se observa que hay algunas especies que aparecen el primer año después de la perturbación y permanecen hasta el final en la mayoría de los cuadrados: Anthyllis vulneraria, Argyrolobium zanonii, Asterolinum linum-stellatum, Fumana ericoides y Ononis minutissima; otras aparecen el segundo año: Avenula bromoides, Eryngium campestre, Euphorbia exigua y Gastridium ventricosum; otra, el tercer año: Crucianella angustifolia; y por último otra, el cuarto año: Aira caryophyllea. Hay un escalonamiento en la germi- 
nación de estas plantas pero la mayoría -11 de las 14 que, generalizando, son 11 de las 42 totales- logran restablecerse sin dificultad. Sólo 3 especies no logran asentarse después de la perturbación: Coronilla minima que aparece el primer y segundo año después de la perturbación y después desaparece, y Blackstonia perfoliata y Bombycilaena erecta que aparecen y desaparecen durante el tercer año. Esta es, de nuevo, otra diferencia de comportamiento con respecto a las plantas oportunistas.

En conclusión podemos decir que dentro de las especies germinadoras existen dos grupos claramente diferenciables en su comportamiento frente a los tratamientos empleados, y que son las especies germinadoras típicas de claros de carrascal o matorrales subseriales, no oportunistas, las realmente adaptadas a este tipo de perturbaciones.

\subsubsection{Comportamiento de las especies rebrotadoras-germinadoras}

El último grupo de plantas según la estrategia de recuperación es el de las rebrotadoras-germinadoras (Tablas 3,4 y 8 , estrategias $5,6,7$ y 8 ). Son plantas que durante los primeros meses después de la perturbación rebrotan; posteriormente el rebrote cede y la estrategia seguida es la germinación. Con este tipo de vida hemos encontrado, en los distintos cuadrados y a lo largo de los años de estudio, 12 especies: 6 de ellas, Erica vagans, Helianthemum canum, Rhamnus alaternus, Silene nutans, Thymus vulgaris y Viola hirta son plantas rebrotadoras mediante órganos aéreos y germinadoras; 1 , Aphyllanthes monspeliensis, es rebrotadora mediante órganos subterráneos y germinadora; 4, Cistus salvifolius, Dorycnium pentaphyllum, Genista hispanica subsp. occidentalis y Genista scorpius son rebrotadoras mediante órganos aéreos y germinadoras, preferentemente germinadoras; y 1, Thesium divaricatum, es rebrotadora mediante órganos aéreos y germinadora, preferentemente rebrotadora.

Resulta evidente la importancia de este grupo de plantas en la recuperación de la vegetación después de los fuegos: muchas de ellas son características del carrascal, aparecen ya desde el primer muestreo, son estables en el tiempo y permanecen hasta el último año (Tabla 8); con su estrategia favorecen, al principio, la regeneración rápida parcial del carrascal, la creación de ambientes favorables para la instalación de otras especies, y después, con su capacidad germinativa, aseguran el mantenimiento de una flora típica básica que haga prosperar la recuperación y la permanencia del carrascal en su conjunto.

\subsection{Evolución de la riqueza florística}

En las Figuras 2, 3 y 4 se representa la riqueza florística de cada cuadrado a lo largo del tiempo: en el eje de abcisas de cada gráfica aparecen las fechas 
EVOLUCIÓN DE LA COMPOSICIÓN FLORÍSTICA POST-FUEGO EN UN CARRASCAL DE NAVARRA

\begin{tabular}{|c|c|c|c|}
\hline Rebrotadores-germinadoras & Estr. & Cuadrados & $\begin{array}{l}\text { Años correlativos } \\
\text { de aparición } \\
\text { en cada cuadrado }\end{array}$ \\
\hline Aphyllanthes monspeliensis & 6 & $\begin{array}{l}\text { B1IS', } \\
\text { B2IS' }\end{array}$ & $\begin{array}{l}1 \\
1 \\
\end{array}$ \\
\hline Cistus salvifolius & 7 & $\begin{array}{l}\text { B1IC, B1IS', } \\
\text { B2IC, B2IS', B2SS' }\end{array}$ & $\begin{array}{l}1234,1234 \\
1234,234,234\end{array}$ \\
\hline Dorycnium pentaphyllum & 7 & $\begin{array}{l}\text { B1IS', B1SS', } \\
\text { B2IC, B2IS', B2SS }\end{array}$ & \begin{tabular}{|l|}
1234,1234, \\
$1234,123,1234$
\end{tabular} \\
\hline Erica vagans & 5 & \begin{tabular}{|l|} 
B1IC, B1IS', \\
B2IS' $^{\prime}$
\end{tabular} & $\begin{array}{l}1234,1234 \\
1234\end{array}$ \\
\hline Genista hispanica ssp. occidentalis & 7 & $\begin{array}{l}\text { B1IC, B1IS', B1SC, } \\
\text { B2IC, B2IS', B2SS' }\end{array}$ & \begin{tabular}{|l|}
$1234,1234,1234$ \\
$1234,1234,34$
\end{tabular} \\
\hline $\begin{array}{l}\text { Genista scorpius } \\
\text { Helianthemum canum }\end{array}$ & $\begin{array}{l}7 \\
5\end{array}$ & $\begin{array}{l}\text { B2IC, B2IS' } \\
\text { B1IS', }^{\prime} \\
\text { B2SC }\end{array}$ & $\begin{array}{l}1234,1234 \\
2 \\
234\end{array}$ \\
\hline Rhamnus alaternus & 5 & B1SS' & 134 \\
\hline Silene nutans & 5 & B2IS' $^{\prime}$ & 1. \\
\hline Thesium divaricatum & 8 & B2IS' $^{\prime}$ & 1234 \\
\hline Thymus vulgaris & 5 & \begin{tabular}{|l|} 
B1SC, \\
B2IS', B2SC
\end{tabular} & \begin{tabular}{|l|}
234, \\
1234,34 \\
\end{tabular} \\
\hline Viola hirta & 5 & $\begin{array}{l}\text { B1IC, B1SS', } \\
\text { B2SC, B2SS' }\end{array}$ & $\begin{array}{l}23,1234 \\
234,1234\end{array}$ \\
\hline
\end{tabular}

Tabla 8. Especies rebrotadoras-germinadoras. Cuadrados y años en que aparecen en cada cuadrado. En negrita las especies presentes en el último año de muestreo y los cuadrados en que permanecen.

Resprouting-germinating species. Plots and years in which they appear in each plot. In bold characters species present in the last year and permanent plots where they remain.

de los muestreos mediante los números consecutivos indicados en el apartado de material y métodos, y en el de ordenadas el número total de especies encontradas en cada cuadrado y en cada muestreo. Se han reunido las distintas gráficas en tres grupos, correspondiendo cada uno al estudio de la composición florística según el tratamiento concreto utilizado.

La evolución de la riqueza florística después de los fuegos controlados sigue el modelo general expuesto y corroborado por otros autores (TRABAUD, 1985, 1990 y 1991b; TRABAUD \& LEPART, 1980 y 1981; PRODON et al., 1984; GARCÍA NOVO, 1977; CASAL et al., 1990; CAÑAS et al., 1990; TÁRREGA \& LUIS, 1990b): después del fuego el suelo queda prácticamente desnudo; a medida que pasa el tiempo se produce un aumento del número 
de especies, que alcanza un punto máximo a partir del cual la riqueza florística tiende a disminuir. Según los autores citados la estabilización de la riqueza florística se alcanza a partir del quinto año, punto al que aún no han llegado nuestras observaciones.

Aún así, en estos cuatro años de estudio hemos podido observar algunas diferencias en la dinámica de la recuperación post-fuego en los distintos cuadrados permanentes que parecen interesantes. Así, el momento en que se alcanza el máximo varía en los distintos cuadrados: en unos se alcanza el primer año después del fuego (B2SS'); en otros, el segundo año (B1IS', B2IC y B2IS'); en dos durante el cuarto año (BISC y B2SC) y, por último, en B1SS' aparecen dos máximos: uno durante el primer año y otro durante el tercero.

En la Figura 2 se observa que en los primeros muestreos después de la perturbación, en los cuadrados de fuego lento (B2) el número de especies es mucho menor que en los de fuego rápido; sin embargo, en los primeros hay un aumento brusco de la riqueza florística en el tercer o cuarto muestreo, llegando a ser ésta siempre mayor a lo largo del tiempo (B2IS' y B2SC) respecto a los de fuego rápido, o bien al menos en algún momento del primer año y principios del segundo (B2IC y B2SS). El tipo de fuego sufrido parece un factor determinante: la perturbación ejercida por el fuego lento es más acusada, y las especies tardan más en regenerarse que en el caso del fuego rápido; sin embargo, al pasar el tiempo el número de especies aumenta y puede ser incluso mayor en caso de fuego rápido. No obstante, hay que tener encuenta dos hechos: primero, el fuego lento se realizó en enero y el primer muestreo en marzo, por lo que quizá este retraso en el fuego haya podido provocar la baja cantidad de especies presentes en los primeros muestreos; y segundo, durante el último año de muestreo las diferencias entre el número de especies presentes en cada pareja de cuadrados tienden a reducirse.

Del análisis de la riqueza florística en función de la situación en la parcela (Figura 3) se deduce que en dos cuadrados inferiores (B1IC y B2IS') la riqueza florística es mayor que en sus homólogos superiores (B1SC y B2SS'); en el resto de los cuadrados inferiores siempre hay algún momento en la evolución en que el número de especies es mayor que en los superiores, bien durante el primer año y principios del segundo (B2IC) o, bien sólo durante la primera parte del segundo año (B1IS'). En cuanto a la situación en la parcela, parece resultar favorecida la recuperación de las zonas bajas; sin embargo, hay que tener en cuenta que las zonas altas del carrascal tienen un grado mayor de pedregosidad, y por lo tanto de escorrentía, condiciones que afectan al rebrote o germinación de algunas especies.

Si atendemos a la riqueza florística según se haya realizado o no adición de suelo no quemado (Figura 4), se observa que durante el primer año (muestreos 1 a 7) y parte del segundo (muestreos 8 a 12) el mayor número 

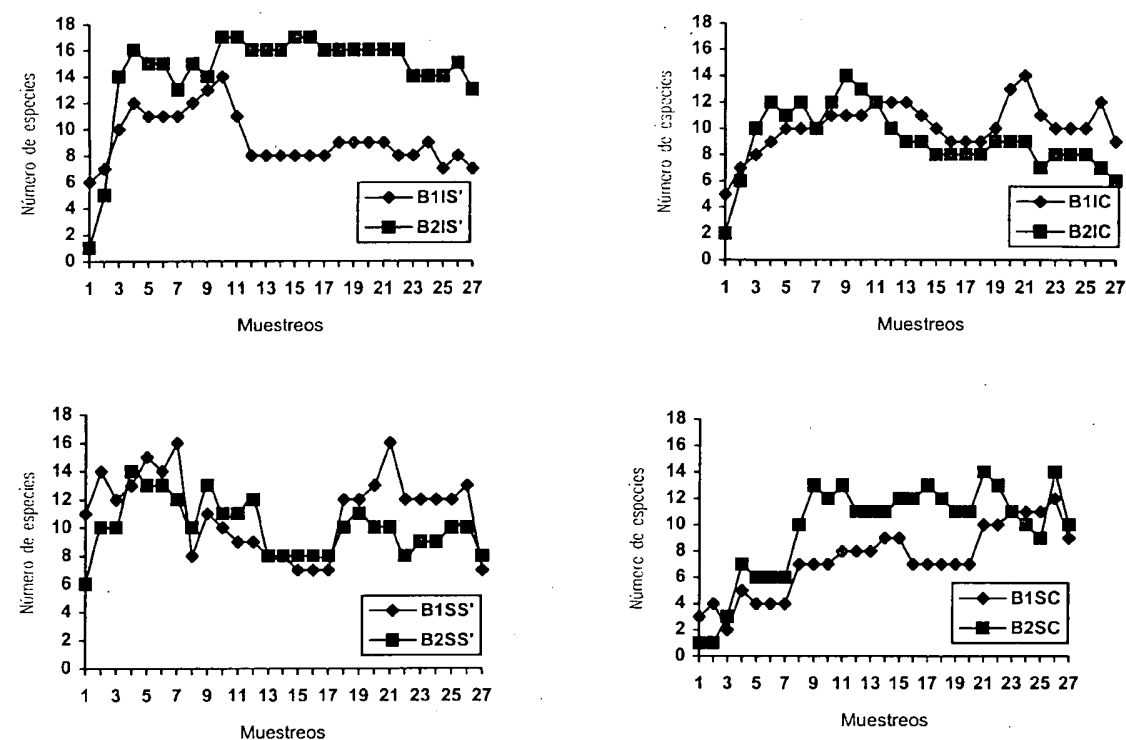

Figura 2. Evolución de la composición florística comparando el tipo de fuego. Floristic composition evolution comparing the type of fire.
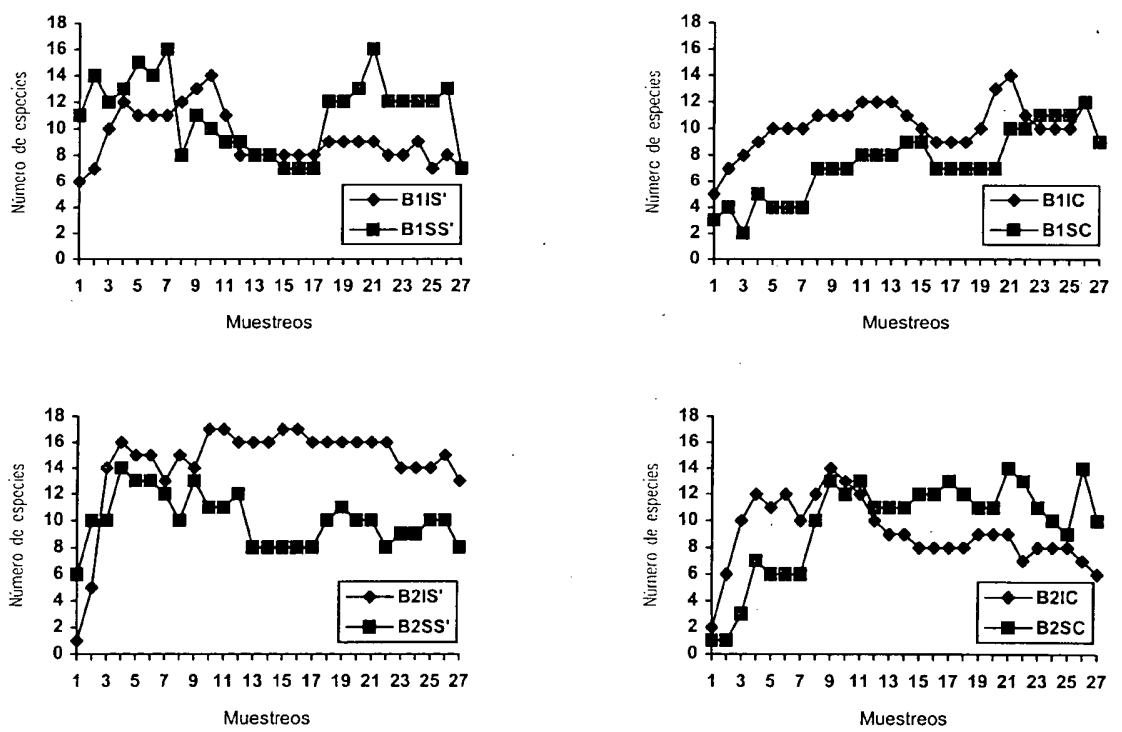

Figura 3. Evolución de la composición florística comparando la situación en la parcela. Floristic composition evolution comparing the situation on the field. 
de especies aparece en los cuadrados sin adición de suelo frente a sus homólogos con adición; también son éstos los que antes alcanzan el máximo de riqueza florística. A partir de la segunda mitad del segundo año de muestreo (muestreos 13 en adelante) el efecto de la adición de suelo no parece tan claro, pues en unos casos la riqueza florística continúa siendo mayor (B2IS' frente a B2IC), en otros se hace menor (B1IS' frente a B1IC) o no muestra una tendencia clara (B1SS' y B2SS' frente a B1SC y B2SC respectivamente). Parece ser que la adición de suelo no quemado es nociva sobre todo en las primeras fases de la recuperación, ya que retarda la regeneración de la vegetación en el carrascal. Esto puede ser debido a que en el carrascal predominan las plantas rebrotadoras, tanto mediante órganos subterráneos como superficiales y al añadir suelo a los cuadrados quedan sepultados y se retarda su regeneración vegetativa; y también a que las semillas de especies oportunistas o no escarificadas por el fuego no emergerán tan fácilmente.

- A partir de los resultados obtenidos en este estudio y de las discusiones a que han dado lugar, creemos muy interesante abordar en el futuro un enfoque complementario de ecología de poblaciones, teniendo en cuenta el núme-
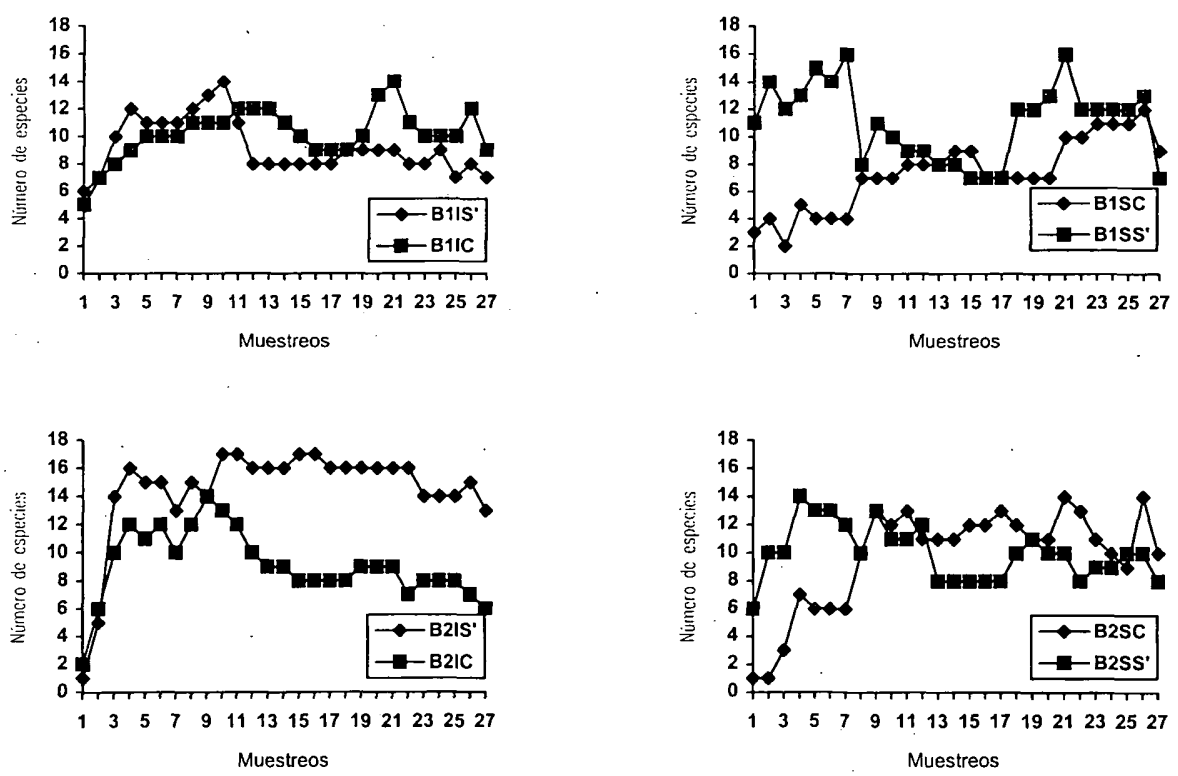

Figura 4. Evolución de la composición florística comparando la adición o no de suelo no quemado. Floristic composition evolution comparing the addition or not of soil not burned. 
ro de individuos de cada especie (frecuencia), lo que permitiría dar a las que se regeneran en mayor número el peso que les corresponde (SILVERTOWN \& LOVETT DOUST, 1993).

\section{Conclusiones}

La composición florística tras fuegos controlados en un carrascal de Navarra tiende a ser igual a la que había antes del fuego, debido al proceso de autosucesión que se da en la vegetación. Las especies del carrascal están adaptadas a este tipo de perturbaciones, pues poseen órganos subterráneos o aéreos que les permiten rebrotar con relativa facilidad. Además aparecen plantas germinadoras de dos tipos: unas oportunistas, que germinan de forma bastante irregular y desaparecen casi siempre antes del cuarto año después de los fuegos, y otras propias de claros y matorrales de sustitución, que germinan con facilidad al crearse calveros tras la perturbación y permanecen cierto tiempo.

La intensidad del fuego afecta a la regeneración en dos aspectos. Por una parte, de forma global, la riqueza florística es mayor en los cuadrados de fuego lento; sin embargo, desde el punto de vista dinámico, a lo largo del tiempo, la riqueza florística de los cuadrados de fuego lento es menor que la de sus homólogos de fuego rápido, especialmente en los primeros meses después de la perturbación. La adición de suelo no quemado es negativa para algunas plantas rebrotadoras porque retrasa la regeneración de la vegetación al colocar las yemas de recambio en una posición no adecuada para que se produzca el proceso de brotación, mientras que para muchas plantas germinadoras es positiva porque aporta semillas extra que logran germinar. Al cabo de un tiempo, 2-3 años después de los fuegos, los efectos de estos tratamientos son menos evidentes.

Agradecimientos. Este trabajo ha sido subvencionado por la CICYT, proyecto FOR90-0928-C0202, y por el Gobierno de Navarra. Agradecemos también los comentarios de los evaluadores, con lo cual el artículo ganó en calidad y claridad. 


\section{Referencias}

ABRAHAMSON, W. G. \& ABRAHAMSON, C. R. (1996). Effects of fire on long-unburned Florida uplands. Journal of Vegetation Science, 7: 565-574.

AFOLAYAN, T. A. (1979). Change in percentage ground cover of perennial grasses under different burning regimes. Vegetatio, 39 (1): 35-41.

AGRAVAL, A. K. (1990). Floristic composition and phenology of temperate grasslands of Western Himalaya as affected by scraping, fire and heavy grazing. Vegetatio, 88: 177-187.

ALLEN, R. B. \& PARTRIDGE, T. R. (1988). Effects of spring and autumm fires on the composition of Chionochloa rigida tussock grassland, New Zealand. Vegetatio, 76: 37-44.

ALMENDROS, G.; MARTÍN, F.; GONZÁLEZ-VILA, F. J. (1988). Effects of fire on humic and lipid fractions in a Dystric Xerochrept in Spain. Geoderma, 42: 115-127.

ARCHIBOLD, O. W. (1979). Buried viable propagules as a factor in postfire regeneration in Northen Saskatchewan. Canadian Journal of Botany, 57: 54-58.

ARNO, S. F.; SMITH, H. Y. \& KREBS, M. A. (1997). Old growthponderosa pineand wester larch stand structures: influences of pre-1900 fires and fire exclusion. USDA Forest Service. Intermountain Research Station. Research paper, 495: 1-21.

BOO, R. M.; PELAEZ, D. V.; BUNTING, S. C.; MAYOR, M. D. \& ELIA, O. R. (1997). Effect of fire on woody species in Central semi-arid Argentina. Journal of Arid Environments, 35: 87-94.

CABEZUDO, B.; PÉREZ LA TORRE, \& A. NIETO, J. M. (1995). Regeneración de un alcornocal incendiado en el Sur de España (Istán, Málaga). Acta Botanica Malacitana, 20: 143-151.

CALVO, L.; TÁRREGA, R. \& LUIS, E. (1993). Use of multivariant analysis to detect post-fire main changes in plant composition in forests of Quercus pyrenaica in Leon province (NW Spain). In: Trabaud, L. \& Prodon, R. (eds), Fire in Mediterranean Ecosystems: 55-68. European Commision. Brussels.

CANADELL, J.; LLORET, F. \& LÓPEZ-SORIA, L. (1991). Resprouting vigour of two mediterranean shrub species after experimental fire treatments. Vegetatio, 95: 119-126.

CAÑAS, J.; LLIMONA, F.; ARRIZABALAGA, A. Y MATHEU, E. (1990). Rehabilitación tras el fuego: el caso de los incendios forestales en Montserrat. Quercus: 12-17.

CARREIRA, J. A. \& NIELL, F. X. (1995). Mobilization of nutrients by fire in a semiarid gorge-crubland ecosystem of Southern Spain. Arid Soil Research and Rehabilitation, 9: 73-89. 
CASAL, M.; BASANTA, M.; GONZÁLEZ, F.; MONTERO, R.; PEREIRAS, J. \& PUENTES, A. (1990). Post-fire dynamics in experimental plots of shrubland ecosystems in Galicia (NW Spain). In J. G. Goldammer \& M. J. Jenkins (eds), Fire in Ecosystems Dynamics: 33-42. The Hague.

CAVERO, R.Y. \& EDERRA, A. (1997a). Evolución de la riqueza florística después de incendios controlados en un robledal de Navarra (España). Pub.Biol.Univ.Navarra, Ser. Bot., 10: 55-66.

CAVERO, R.Y. \& EDERRA, A. (1997b). Recuperación de la vegetación después de incendios controlados en un carrascal de Navarra: Evolución de la cobertura en los tres años siguientes a la perturbación. Actas del I Congreso Forestal hispano-luso, Irati 97, Pamplona, mesa 2: 241-246.

CLEMENTE, A. S.; REGO, F.C. \& CORREIA, O. A. (1996). Demographic patterns and productivity of post-fire regeneration in Portuguese Mediterranean Maquis. Int. Journal Wildland Fire, 6 (1): 5-12.

COCKS, M. P. \& STOCK, W. D. (1997). Heat stimulated germination in relation to seed characteristics in fynbos legumes of the Western Cape Province, South Africa. South African Journal of Botany, 63 (3): 129-132.

COLLINS, S. L. (1989). Experimental analysis of patch dynamics and community heterogeneity in tallgrass prairie. Vegetatio, 85: 57-66.

DE LILLIS, M. \& TESTI, A. (1990). Post-fire dynamics in a disturbed mediterranean community in central Italy. In J. G. Goldammer \& M. J. Jenkins (eds). Fire in Ecosystem Dynamics: 53-62. The Hague.

EDERRA, A.; URDÍROZ, A. \& CAVERO, R. Y. (1997). Floristic richness evolution after controlled fires in two woods of Navarra (Spain). Lagascalia, 19 (1-2): 769-776.

FENNER, M., ED. (1992). Seeds. The Ecology of Regeneration in Plant Communities. CAB International, Oxon, UK. 373 pp.

FERNÁNDEZ, I.; CABANEIRO, A. \& CARBALLAS, T. (1997). Organic matter changes inmediately after a wildfire in an atlantic forest soil and comparison with laboratory soil heating. Soil Biol. Biochem., 29 (1): 1-11.

FRANKLIN, S. B.; ROBERTSON, P. A. \& FRALISH, J. S. (1997). Small-scale fire temperature patterns in upland Quercus communities. Journal of Applied Ecology, 34: 613-630.

GARCÍA FAYOS, P. (1991). La vegetación silicícola de la Sierra Calderona (Comunidad Valenciana). Lazaroa, 12: 317-322.

GARCÍA NOVO, F. (1977). The effects of fire on the vegetation of Donana National Park, Spain. Enviromental in Mediterranean Ecosystems. USDA Forest Service General Technical Report WO 3: 318-325.

GÓMEZ GUTIÉRREZ, J. M.; GONZÁLEZ BARTOLOMÉ, R.; FERNÁNDEZ SANTOS, B. \& GALINDO VILLARDÓN, P. (1988). Regeneración postfuego del piornal serrano. Formaciones de Cytisus balansae (Boiss) Ball. Anu. Cent. Edafol. Biol. Apl. Salamanca, 13: 261-277. 
GONZÁLEZ-RABANAL, F. \& CASAL, M. (1995). Effects of high temperatures and ash on germination of ten species from gorse shrubland. Vegetatio, 116: 123-131.

GRATANI, L. \& AMADORI, M. (1991). Post-fire resprouting of shrubby species in mediterranean maquis. Vegetatio, 96: 137-143.

GRAY, A. N. \& FRANKLIN, J. F. (1997). Effects of multiple fires on the structure of Southwestern Washington forests. Northwest Science, 71 (3): 174-185.

HANLEY, M. E. \& FENNER, M. (1997). Seedling growth of four fire-following mediterranean plant species deprived of single mineral nutrients. Functional Ecology, 11: 398-405.

HURLBERT, S. H. (1984). Pseudoreplication and design of ecological field experiments. Ecological Monographs, 54(2): 187-211.

KEELEY, J. E. (1987). Role of fire in seed germination of woody taxa in California chaparral. Ecology, 68: 434-443.

KEELEY, J. E. (1991). Seed germination and life history sindromes in the California chaparral. The Botanical Review, 57: 81-116.

KEELEY, J. E. (1992). Demographic structure of California chaparral in the long-term absence of fire. Journal of Vegetation Science, 3: 79-90

KEELEY, J. E. \& FOTHERINGHAM, C. J. (1998). Smoke-induced seed germination in California chaparral. Ecology, 79 (7). 2320-2336.

KEELEY, J. E.; KEELEY, M. B. \& BOND, W. J. (1999). Stem demography and postfire recruitment of a resprouting serotinous conifer. Journal of Vegetation Science, 10: 69-76.

KRUGER, F. J. (1983). Plant community diversity and dynamics in relation to fire. In: Kruger, F.J., Mitchell, D. T. \& Jarvis, J. U. M. (Eds), MediterraneanType Ecosystems. The Role of Nutrients: 446-472. Springer-Verlag. Berlin.

KUTIEL, P. \& KUTIEL, H. (1989). Effects of a wildfire on soil nutrients and vegetation in an Aleppo pine forest on Mount Carmel, Israel. Pirineos, 134: 59-74.

LATERRA, P. (1997). Post burn recovery in the flooding pampa: impact of an invasive legume. Journal Range Manage, 50:-274-277.

LLORET, F. \& LÓPEZ-SORIA, L. (1993). Resprouting of Erica multiflora after experimenta fire treatments. Journal of Vegetation Science, 4: 367-374.

LLORET, F. \& VILA, M. (1997). Clearing vegetation in mediterranean garrigue: response after a wildfire. Forest Ecology and Management, 93:227-234.

LLORET, F. \& ZEDLER, P. H. (1991). Recruitment pattern of Rhus integrifolia populations in periods between fire in Chaparral. Journal of Vegetation Science, 2: 217-230.

MALLIK, A. U. \& GIMINGHAM, C. H. (1983). Regeneration of heathland following burning. Vegetatio, 53: 45-58.

MARTÍNEZ-SÁNCHEZ, J. J.; MARIN, A.; HERRANZ, J. M.; FERRANDIS, P. \& DE LAS HERAS, J. (1995). Effects of high temperatures on germination 
of Pinus halepensis Mill and Pinus pinaster Aiton ssp. pinaster seeds in Southeast Spain. Vegetatio, 116: 69-72.

MAZZOLENI, S. (1989). Fire and Mediterranean Plants: Germination responses to heat exposure. Annali di Botanica, 47: 227-233.

MORENO, J. M. \& OECHEL, W. C. (1994). Fire intensity as a determinant factor of post-fire plant recovery in Southern California Chaparral. In: Moreno, J. M. \& Oechel, W. C. (eds.), The role of fire in Mediterranean-Type ecosystems: 26-45 Springer-Verlag. New York.

OJEDA, F.; MARAÑON, T. \& ARROYO, J. (1996). Postfire regeneration of a mediterranean heatland in Southdern pain. Int. Journal Wildland Fire, 6 (4): 191-198.

PAPIÓ, C. (1988). Respuesta al fuego de las principales especies de la vegetación de Garraf (Barcelona). Orsis, 3: 87-103.

PERY, M. (1989). Observacions sobre la regeneració de les suredes després del foc. Scientia Gerundensis, 15: 181-184.

PICKETT, S. T. A. \& WHITE, P. S. (1985). The Ecology of Natural Disturbance and Patch Dynamics. Academic Press, Orlando, Fl. USA.

PICKETT, S. T. A.; KOLASA, J.; ARMESTO, J. J. \& COLLINS, S. L. (1989). The ecological concept of disturbance and its expression at various hierarchical levels. Oikos, 54: 129-136.

PONS, T. L. (1992): Seed responses to light. In: Fenner, M. (ed.), Seeds: The ecology of regeneration in plant communities: 259-284. CAB International, Oxon, UK.

POSSINGHAM, P. H.; CONNINS, H. N. \& NOBLE,I. R. (1995). The fire and flammability niches in plant communities. Journal of Theoretical Biology, 174: 97-108.

PRODON, R.; FONS, R. \& PETER, A. M. (1984). L'impact du feu sur la vegetation, les oiseaux et les micromammifères dans diverses formations méditerranéennes des Pyrénées orientales: premiers résultats. Revue d'Ecologie, Terre et Vie 39: 129-158.

SARACINO, A.; PACELLA, R.; LEONE, U. \& BORGHETTI, M. (1997). Seed dispersal and changing seed characteristics in a Pinus hälepensis Mill. forest after fire. Plant Ecology, 130:13-19.

SCHAEFER, J. A. (1993). Spatial patterns in Taiga plant communities following fire. Canadian Journal of Botany, 71: 1568-1573.

SCHAETZL, R. J. (1994). Changes in 0 horizon mass, thickness and carbon content following fire in Northern hardwood forests. Vegetatio, 115: 41-50.

SIEG, C. H. \& WRIGHT, H. A. (1996). The role of prescribed burning in regenerating Quercus macrocarpa and associated woody plants in stringer woodlands in The Black Hills, South Dakota. Int. Journal Wildland Fire, 6(1): 21-29.

SILVERTOWN, J. W. \& LOVETT DOUST, J. (1993). Introduction to Plant Population Biology. 3rd. ed. Blackwell Science Ltd. Oxford. 210 pp. 
SINGH, R. S. (1993). Effect of winter fire on primary productivity and nutrient concentration of a dry tropical savanna. Vegetatio, 106: 63-71.

SOUTO, C. \& MONTEIRO, A. (1987). Ecological fire influences on Quercus suber forest ecosystems. Ecologia Mediterranea, 13 (4): 69-77:

TÁRREGA, R. \& LUIS, E. (1988a). Sucesión post-fuego y mecanismos de supervivencia en comunidades de Quercus pyrenaica en la provincia de León. II Jornadas sobre bases ecológicas para la gestión ambiental. Zaragoza.

TÁRREGA, R. \& LUIS, E., (1988b). Estudio comparativo de la composición florística anterior y posterior al fuego en robledales de Quercus pyrenaica de la provincia de León. VIII Jornadas de Fitosociología. Málaga. 105-106.

TÁRREGA, R. \& LUIS, E. (1990a). La problemática de los incendios forestales y su incidencia sobre los robledales de Quercus pyrenaica en la provincia de León. Ecología, fuera de serie n ${ }^{\circ}$ 1: 223-237.

TÁRREGA, R. \& LUIS, E. (1990b). Forest fires and climatic features in León province (Spain)- Fire effects on Quercus pyrenaica ecosystems. In J. G. Goldammer \& M. J. Jenkins (Eds.), Fire in Ecosystem Dynamics: 63-69. The Hague.

TÁRREGA, R.; CALVO, R. \& LUIS, E. (1990). Estudio comparativo de la composición florística en la regeneración post-fuego en ecosistemas de Quercus pyrenaica Wild. Acta Botanica Malacitana, 15: 331-339.

TARREGA, R.; LUIS, E. \& MARCOS, E. (1996). Relationship Between soil changes and plant succession in post-fire regeneration of Quercus pyrenaica ecosystems. Arid Soil Research and Rehabilitation, 10: 85-93.

THANOS, C. A. DASKALAKOV, E. N. \& NIKOLAIDOV, S. (1996). Early post-fire regeneration of a Pinus halepensis forest on Mount Párnis, Greece. Journal of Vegetation Science, 7: 273-280.

THOMPSON, K. (1992). The functional ecology of seed banks. In: Fenner, M. (ed.), Seeds: The ecology of regeneration in plant communities: 231-258. CAB International, Oxon, UK.

TRABAUD, L. (1985). Aspect floristique de la recolonization des garrigues de Quercus coccifera et des forêts de Pinus halepensis après incendie in Bas Languedoc (Sudde la France). Jornadas sobre bases ecológiques per la gestió ambiental: 13-16. Montesquiu (Ripollès).

TRABAUD, L. (1987). Fire and survival traits of plants. In: Trabaud L. (ed.), The role of fire ecological systems: 65-89. Academic Publishing. The Hague.

TRABAUD, L. (1990). Fire resistence of Quercus coccifera L. garriga. In: Goldammer, J. G. \& Jenkins, M. J. (eds), Fire in Ecosystems Dynamics: 21-32. The Hague.

TRABAUD, L. (1991a). Fire regimes and phytomas growth dynamics in a Quercus coccifera garrigue. Journal of Vegetation Science 2: 307-314.

TRABAUD, L. (1991b). Le feu est-il un facteur du changement pour les systèmes écologiques du bassin méditerranéen? Sécheresse 3: 163-174. 
TRABAUD, L. (1994). The effect of fire on nutrient losses and cycling in a Quercus coccifera garrigue (Southern France). Oecologia, 99: 379-386.

TRABAUD, L. \& CHANTERAC, B. (1985). The influence of fire on the phenological behaviour of mediterranean plant species in Bas-Languedoc (Southern France). Vegetatio, 60: 119-130.

TRABAUD, L. \& LEPART, J. (1980). Diversity and stability in garriga ecosystems after fire. Vegetatio 43: 49-57.

TRABAUD, L. \& LEPART, J. (1981). Changes in the floristic composition of a Quercus coccifera L. garrigue in relation to different fire regimes. Vegetatio 46: 105-116.

TRABAUD, L.; MARTÍNEZ-SÁNCHEZ, J. J.; FERRANDIS, P.; GONZÁLEZOCHOA, A. I. \& HERRANZ, J. M. (1997). Végétation épigée et banque de semences du soil: leur contribution á la stabilité ciclique des pinédes mixtes de Pinus halepensis et Pinus pinaster. Canadian Journal of Botany, 75: 10121021.

TUTIN, T. G., HEYWOOD, V. H. et al. (1964-1980). Flora Europaea. 5 Vols. Cambridge University Press. Cambridge.

URDIROZ, A. (1999). Estudio del impacto de los incendios forestales en la brioflora y su recuperación. Tesis Doctoral. Universidad de Navarra. 541 pp.

WHITTLE, C. A.; DUCHESNE, L. C. \& NEEDHAM, T. (1997). The impact of broadcast burning and fire severity on species composition and abundance of surface vegetation in a Jack pine (Pinus banksiana) clear-cut. Forest Ecology and Management, 94: 141-148.

WILLIAMS, P. R. \& CLARKE, P. J. (1997). Habitat segregation by serotindus shrubs in heaths: post fire emergence and seedling survival. Australian Journal of Botany, 45: 31-39.

WILLSON, M. F.. (1992). The ecology of seed dispersal. In Fenner, M. (ed.), Seeds: The ecology of regeneration in plant communities: 61-85. CAB International, Oxon, UK. 


\section{Anexo}

Los resultados obtenidos en cuanto a la presencia de especies a lo largo del tiempo aparecen en la Tabla 3. La tabla está dividida en ocho partes, cada una correspondiente a los resultados de cada uno de los cuadrados permanentes. Los taxa están ordenados teniendo en cuenta su orden de aparición después de los fuegos y su permanencia en cada cuadrado. Cada columna representa un muestreo; la cifra superior de la columna es el número asignado a cada muestreo (según se ha indicado en el apartado de material y métodos), mien-
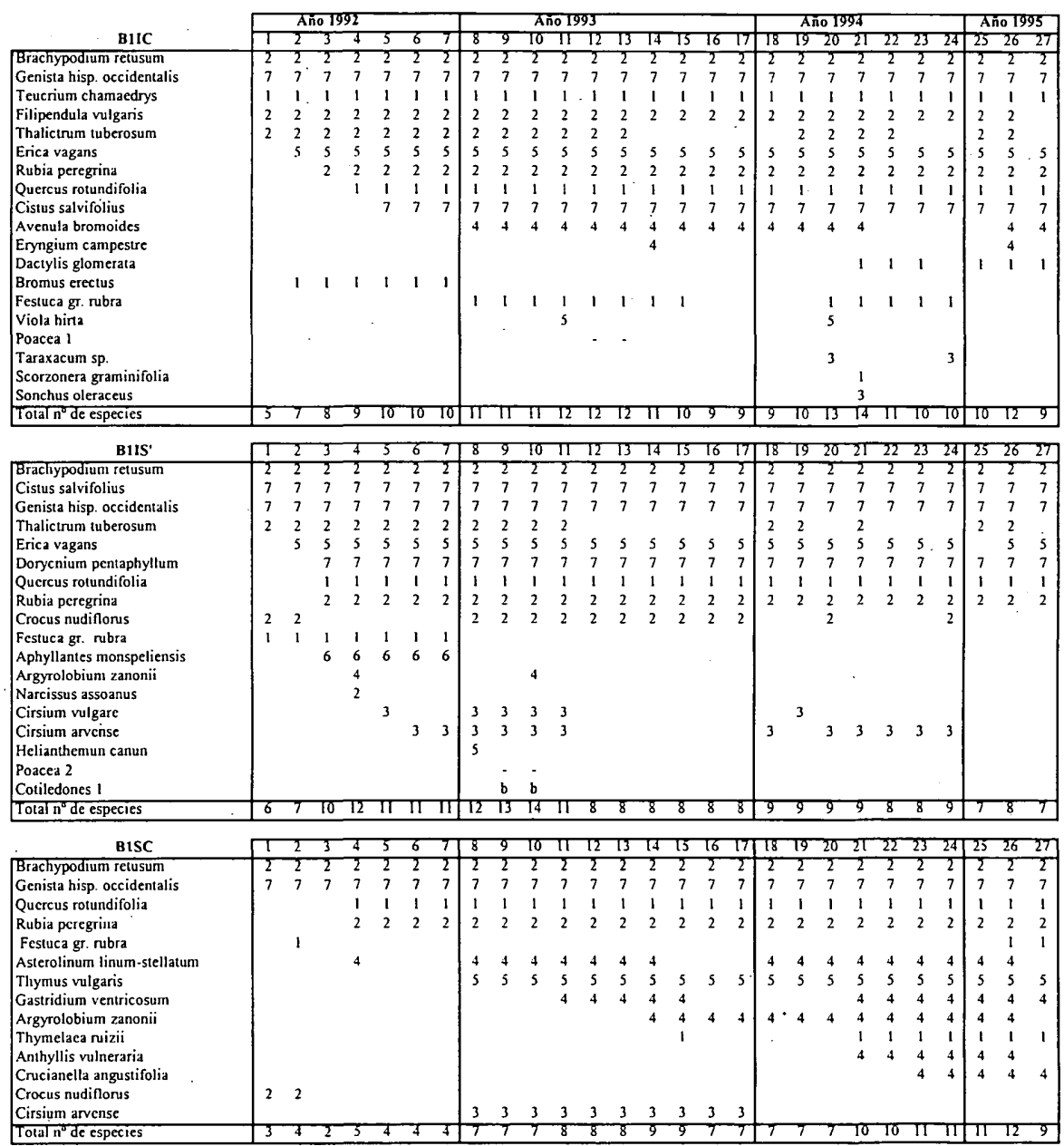

90 
EVOLUCIÓN DE LA COMPOSICIÓN FLORÍSTICA POST-FUEGO EN UN CARRASCAL DE NAVARRA

\begin{tabular}{|c|c|c|c|c|c|c|c|c|c|c|c|c|c|c|c|c|c|c|c|c|c|c|c|c|c|c|c|}
\hline \multirow[b]{2}{*}{ B1SS' } & \multicolumn{7}{|c|}{ Año 1992} & \multicolumn{10}{|c|}{ Anio 1993} & \multicolumn{7}{|c|}{ Año 1994} & \multicolumn{3}{|c|}{ Año 1993 } \\
\hline & $T$ & 2 & 3 & & 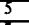 & 6 & 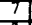 & 8 & & & & & 13 & 14 & 15 & & 17 & 18 & & & & 22 & & & & & \\
\hline Brachypodium relusum & 2 & $\sqrt{2}$ & 2 & 2 & 2 & 2 & 2 & 2 & 2 & 2 & 2 & 2 & 2 & 2 & 2 & $?$ & 2 & 2 & 2 & 2 & & 2 & & & & & \\
\hline Dorycrium pentaphyllum & 7 & 77 & 7 & 7 & 7 & 7 & 7 & 7 & 7 & 7 & 7 & 7 & & 7 & 7 & & 7 & 7 & & 7 & & 7 & & & & & \\
\hline Rubia peregrina & 2 & 2 & 2 & 2 & 2 & 2 & 2 & 2 & 2 & 2 & 2 & 2 & & 2 & 2 & 2 & 2 & 2 & & 2 & 2 & 2 & & & & & \\
\hline Viola hirta & 5 & 55 & 5 & 5 & 5 & 5 & 5 & 5 & 5 & 5 & 5 & 5 & 5 & 5 & 5 & 5 & 5 & 5 & 5 & 5 & 5 & 5 & 5 & 5 & 55 & 5 & \\
\hline Cirsium vulgare & 3 & 3 & 3 & 3 & 3 & 3 & 3 & 3 & 3 & 3 & 3 & 3 & & 3 & 3 & 3 & 3 & & 3 & 3 & 3 & 3 & & & & & \\
\hline Carex hallerana & 1 & 1 & 1 & 1 & 1 & 1 & 1 & & & & & & & I & 1 & 1 & 1 & 1 & 1 & 1 & 1 & 1 & & & & & 1 \\
\hline Festuca gr. rubra & 1 & 11 & 1 & 1 & 1 & 1 & 1 & & & & & & & & & & & & & 1 & 1 & 1 & & 1 & 11 & 1 & \\
\hline Asterolinum linum-stellatum & & 44 & 4 & 4 & 4 & 4 & 4 & 4 & 4 & 4 & 4 & 4 & 4 & 4 & & & & 4 & 4 & 4 & 4 & 4 & & 4 & 4 & 4 & \\
\hline Quercus rotundifolia & & & 1 & 1 & 1 & 1 & 1 & 1 & 1 & 1 & 1 & 1 & 1 & 1 & 1 & 1 & 1 & 1 & I & 1 & 1 & 1 & 1 & 1 & 11 & 1 & 1 \\
\hline Rhamnus alaternus & & & & 5 & 5 & 5 & 5 & & & & & & & & & & & & 5 & & & & & & & & \\
\hline Anthyllis vulneraria & & & & & 4 & 4 & 4 & 4 & 4 & 4 & 4 & 4 & 4 & & & & & & & & & & & & & 4 & \\
\hline Potentilla tabernaemontani & & & & & 1 & 1 & & & 1 & 1 & & & & & & & & 1 & $\mathbf{I}$ & 1 & 1 & 1 & 1 & & 1. 1 & 1 & \\
\hline $\begin{array}{l}\text { Cirsium arvense } \\
\text { Euphorbia exiqa }\end{array}$ & & & & & & & & & & & & & & & & & & & & & 3 & 3 & 3 & & $3: 3$ & 3 & \\
\hline \begin{tabular}{|l} 
Euphorbia exigua \\
Conopodium majus
\end{tabular} & & 22 & 2 & 2 & 2 & 2 & & & 2 & 2 & 2 & & & & & & & & & 2 & $\begin{array}{l}4 \\
2\end{array}$ & & & & & & \\
\hline Filipendula vilgaris & & & & & 2 & 2 & 2 & & & 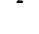 & 2 & & & & & & & & 2 & 2 & 2 & & & & & & \\
\hline Cotiledones 4 & & & & & & & & & & & & & & & & & & & & & & & & & & & \\
\hline $\begin{array}{l}\text { Sonchus oleraceus } \\
\text { Fritillaria pyrenaica }\end{array}$ & 3 & 33 & 3 & 3 & 3 & 3 & .3 & & & & & 3 & & & & & & & & & & & & & & & \\
\hline $\begin{array}{l}\text { Fritillaria pyrenaica } \\
\text { Crocus nudiflorus }\end{array}$ & & $\begin{array}{l}22 \\
2\end{array}$ & 2 & 2 & & & & & & & & & & & & & & & & & & & & & & & \\
\hline Cotiledones 2 & & b & & & & & & & & & & & & & & & & & & & & & & & & & \\
\hline $\begin{array}{l}\text { Poacea } 3 \\
\text { Ononis m }\end{array}$ & & & & & 4 & & & & & & & & & & & & & & & & & & & & & & \\
\hline $\begin{array}{l}\text { Rhamnus saxatilis } \\
\text { Rhasima }\end{array}$ & & & & & 4 & & 1 & & & & & & & & & & & & & & & & & & & & \\
\hline Cotiledones & & & & & & & b & & & & & & & & & & & & & & & & & & & & \\
\hline $\begin{array}{l}\text { Poacea } 4 \\
\text { Blackstonia perfoliata }\end{array}$ & & & & & & & & & $\cdot$ & & & & & & & & & & & & 4 & & & & & & \\
\hline $\begin{array}{l}\text { Blackstonia perfoliata } \\
\text { Total } n^{\circ} \text { de especies }\end{array}$ & $\pi$ & $14 \mathrm{~T}$ & 12 & 13 & 15 & 14 & 16 & 8 & $\pi$ & 10 & 9 & 9 & 8 & 8 & 7 & 7 & 7 & 12 & 12 & 73 & 16 & 12 & 121 & & 121 & $\sqrt{3}$ & \\
\hline B2IC & $T$ & 7 & & 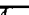 & 5 & & & 8 & & TO & & & & & & & & & & & & & & & & & \\
\hline Genista hisp. occidentalis & $\frac{1}{7}$ & 23 & $\frac{3}{7}$ & 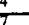 & $\frac{3}{7}$ & 7 & 7 & $\frac{8}{7}$ & $\frac{y}{7}$ & $\frac{10}{7}$ & $\frac{11}{7}$ & $\frac{12}{7}$ & 13 & $\frac{14}{7}$ & $\frac{13}{7}$ & $\frac{10}{7}$ & 7 & $\frac{78}{7}$ & $\frac{19}{7}$ & $\frac{200}{7}$ & $\frac{21}{7}$ & $\frac{22}{7}$ & $\frac{2325}{7}$ & t4 & $\frac{2024}{7}$ & 82 & \\
\hline $\begin{array}{l}\text { Brachypodium retusum } \\
\text { Buspos }\end{array}$ & & 22 & 2 & 2 & 2 & 2 & 2 & 2 & 2 & 2 & 2 & 2 & 2 & & 2 & 2 & 2 & 2 & 2 & 2 & 2 & 2 & & 2 & 22 & & \\
\hline Cistus salvifolius & & & 7 & 7 & 7 & 7 & 7 & 7 & 7 & 7 & 7 & 7 & 7 & & 7 & & 7 & & 7 & 7 & 7 & 7 & & & 77 & 77 & \\
\hline Galium fruticescens & & 11 & 1 & 1 & 1 & 1 & 1 & & 1 & 1 & 1 & 1 & 1 & 1 & 1 & & & 1 & 1 & 1 & 1 & 1 & & & 11 & & \\
\hline Dorycnium pentaphyllum & & & 7 & 7 & 7 & 7 & 7 & 7 & 7 & 7 & 7 & 7 & 7 & 7 & 7 & 7 & 7 & 7 & 7 & 7 & 7 & 7 & 7 & 7 & 77 & 77 & \\
\hline Quercus rotundifolia & & & 1 & I & 1 & 1 & 1 & I & 1 & 1 & 1 & 1 & 1 & 1 & 1 & & 1 & 1 & 1 & 1 & 1 & 1 & 1 & & 11 & 11 & \\
\hline Argyrolobium zanonii & & & & & 4 & 4 & 4 & 4 & 4 & 4 & 4 & 4 & 4 & & 4 & & & & 4 & 4 & 4 & & & & 4 & & \\
\hline Genista scorpius & & & & & & 7 & & & & 7 & 7 & 7 & 7 & 7 & 7 & & & 7 & 7 & 7 & 7 & 7 & & & & & \\
\hline & 1 & & & & & & & & & & & & & & & & & & & & & & & & & & \\
\hline Thalictrum tuberosur & & 2 & 2 & 2 & 2 & 2 & & 2 & 2 & 2 & 2 & 2 & & & & & & & 2 & & 2 & & & & & & \\
\hline Narcissus assoanus & & 22 & 2 & 2 & & & & & 2 & & & & & & & & & & & & & & & & & & \\
\hline Picris echioides & & & 3 & 3 & 3 & 3 & ${ }^{3}$ & & & & & & & & & & & & & & & & & & & & \\
\hline $\begin{array}{l}\text { Cotiledones } 4 \\
\text { Anthericum liliago }\end{array}$ & & & & & & & & b & & & & & & & & & & & & & & & & & & & \\
\hline $\begin{array}{l}\text { Anthericum liliago } \\
\text { Ptlomis lychnitis }\end{array}$ & & & 2 & I & 1 & I & 1 & 1 & 1 & 1 & 1 & 1 & 1 & 1 & & & & & & & & & & & & & \\
\hline $\begin{array}{l}\text { Palomis lychn } \\
\text { Sonchus asper }\end{array}$ & & & & 3 & & 3 & 3 & 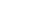 & & & & & & & & & & & & & & & & & & & \\
\hline $\begin{array}{l}\text { Sonchus asper } \\
\text { Muscari neglectum }\end{array}$ & & & & & & & & & 2 & 2 & 2 & & & & & & & & & & & & & & & & \\
\hline Asterolinum linum-s & & & & & & & & 4 & 4 & 4 & .4 & & & & & & & & & & & & & & & & \\
\hline Crocus nudiflorus & & & & & & & & 2 & 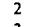 & 3 & & & & & & & & & & & & & & & & & \\
\hline $\begin{array}{l}\text { Picris hieracioides } \\
\text { Total } n^{\circ} \text { de especies }\end{array}$ & & $\begin{array}{lll}6 & 0\end{array}$ & 10 & $\sqrt{12}$ & $\pi$ & 12 & Tण & 12 & 14 & $\frac{3}{13}$ & $\sqrt{12}$ & 10 & 5 & 9 & 8 & 8 & 87 & 8 & 9 & 9 & 9 & & 8 & 8 & 8 & 76 & 6 \\
\hline
\end{tabular}


PIRINEOS 153-154

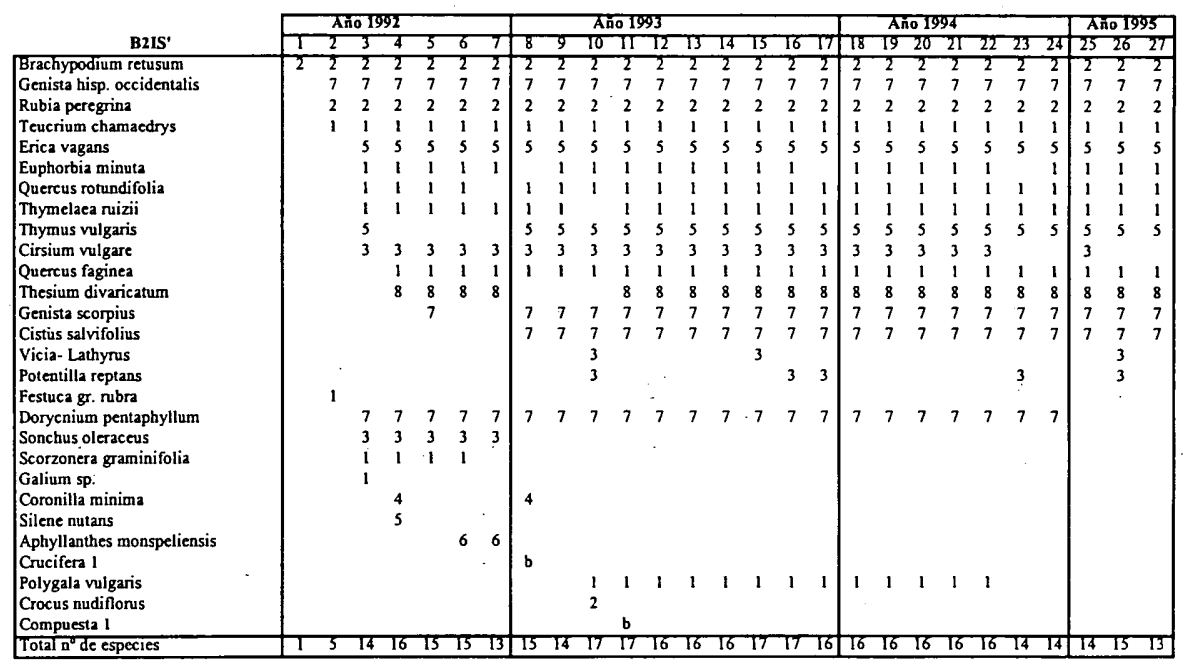

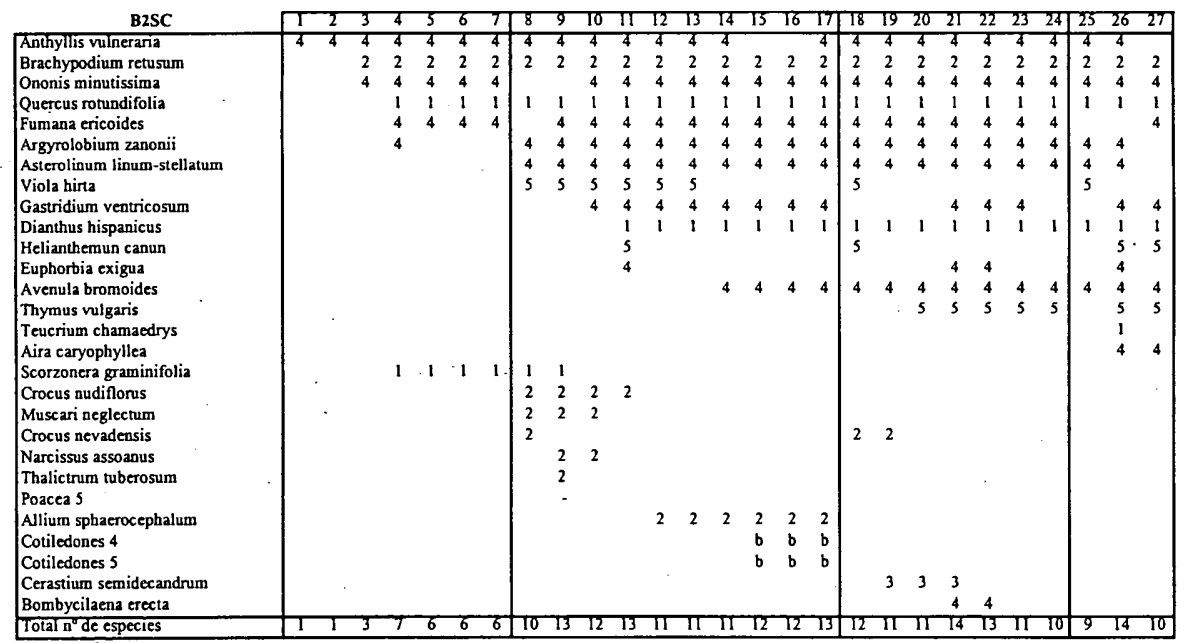

92 


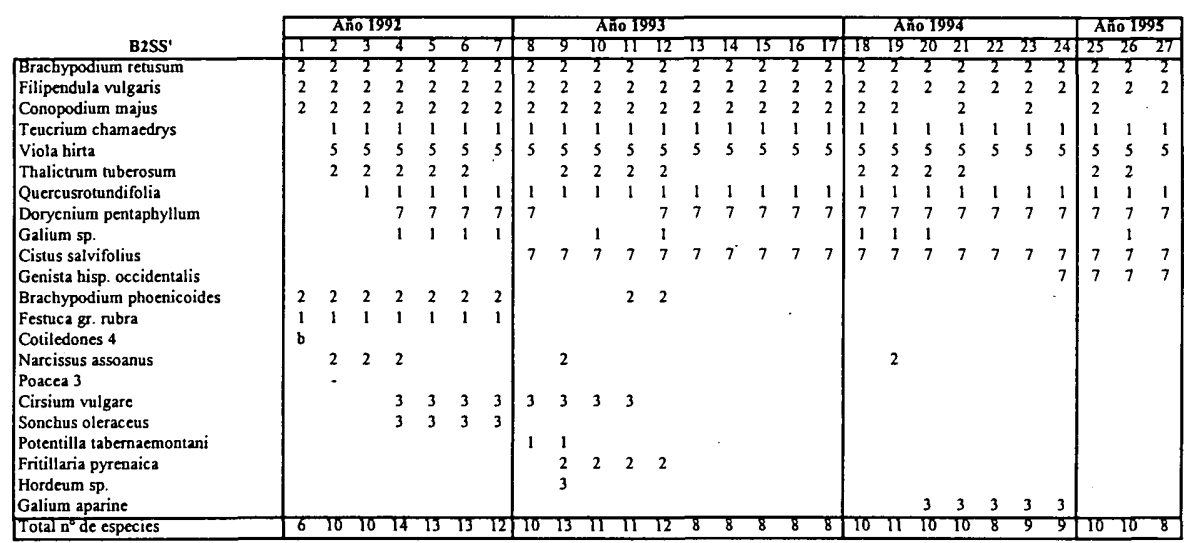

1, plantas rebrotadoras mediante órganos superficiales; 2, plantas rebrotadoras mediante órganos subterráneos; 3, plantas germinadoras oportunistas; 4, plantas germinadoras no oportunistas; 5, plantas rebrotadoras mediante órganos superficiales y germinadoras; 6, plantas rebrotadoras mediante órganos subterráneos y germinadoras; 7 , plantas rebrotadoras mediante órganos superficiales y germinadoras, preferentemente germinadoras; 8 , plantas rebrotadoras mediante órganos superficiales y germinadoras, preferentemente rebrotadoras; $b$, plantas germinadoras; -, estrategia desconocida.

Tabla 3. Evolución de la composición florística en los cuadrados permanentes. Floristic composition evolution in the permanent plots.

tras que la última es el número total de especies que aparece en el muestreo correspondiente. La presencia de cada especie se ha señalado en la tabla mediante un número que alude a su estrategia de regeneración. El pie de la tabla nos muestra la significación de cada uno de estos números que aluden a la estrategia de regeneración de la especie en el rodal.

En la Tabla 4 figuran todos los taxa que han aparecido a lo largo de los cuatro años de estudio, tanto en las parcelas quemadas como en la zona control sin quemar o en toda el área antes de provocar los fuegos. Se indica en esta tabla, para cada especie, en la primera columna, el tiempo en que se ha encontrado la especie; en la segunda en qué tipo de parcelas han aparecido; en la tercera columna se indica la estrategia regeneradora que es la misma en todas las combinaciones de tratamientos; en la cuarta columna figura el número total de cuadrados permanentes en que se ha desarrollado cada taxa; en la quinta columna el número de cuadrados permanentes en los que aparece cada taxa al final del estudio; la sexta columna informa en qué años del estudio ha aparecido cada especie; por último, en la séptima columna se indican los cuadrados permanentes en que han aparecido en cualquier momento a lo largo del estudio. Cuando los taxa se han encontrado tanto en los cuadrados de la parcela quemada con fuego rápido como en los de la quemada con 
fuego lento, se indica entre paréntesis (columnas cuarta y quinta) el número de cuadrados de cada tipo, siendo siempre el primer número el correspondiente al fuego rápido (B1) y el segundo al fuego lento (B2). Se resalta en negrita todos los datos de los taxa que, estando presentes el último año de estudio, han mantenido su presencia en todos los cuadrados permanentes en que en algún momento comenzaron a desarrollarse. También se resalta en negrita el número 4, para indicar qué taxa son los que forman parte de la vegetación a los cuatro años de ocurrir las perturbaciones. Con fondo oscuro destacamos los taxa que han aparecido sólo después de las perturbaciones; lógicamente, coinciden con los que siguen una estrategia de regeneración de tipo 3: germinadoras oportunistas. Los taxa que no se han podido identificar al menos a nivel genérico aparecen al final de la tabla 4. En las últimas casillas de la tabla se da el número total de especies de cada tipo.

\begin{tabular}{|c|c|c|c|c|c|c|c|}
\hline Taxa & Tiem: & Fueg. & Estr. & $\begin{array}{l}\text { Tot. } \\
\text { cuad. }\end{array}$ & $\begin{array}{l}\text { Final. } \\
\text { cuad. }\end{array}$ & Años & Cuadrados \\
\hline Aira caryophyllea & AD & B2 & 4 & 1 & 1 & 4 & B2SC \\
\hline Allium sphaerocephalum & $\mathrm{AD}$ & $\mathrm{B} 2$ & 2 & 1 & 0 & 2 & $\mathrm{~B} 2 \mathrm{SC}$ \\
\hline Amelanchier ovalis & A & & & & & & \\
\hline Anacamptis pyramidalis & $\overline{\mathrm{A}}$ & & & & & & \\
\hline Anthericum liliago & $\mathrm{AD}$ & $\mathrm{B} 2$ & 2 & 1 & 0 & 1 & B2IC \\
\hline Anthoxanthum odoratum & $\overline{\mathrm{A}}$ & & & & & & \\
\hline Anthyllis vulneraria & AD & B1B2 & 4 & $3(2,1)$ & $3(2,1)$ & 1234 & $\begin{array}{l}\text { B1SC, } \\
\text { B1SS', } \\
\text { B2SC }\end{array}$ \\
\hline Aphanes arvensis & $\overline{\mathrm{A}}$ & & & & & & \\
\hline Aphyllanthes monspeliensis & AD & B1B2 & 6 & $2(1,1)$ & 0 & 1 & $\begin{array}{l}\text { B1IS', }^{\prime} \\
\text { B2IS' }^{\prime}\end{array}$ \\
\hline Arctostaphyllos uva-ursi & $\mathrm{A}$ & & & & & & \\
\hline Argyrolobium zanonii & $A D$ & B1B2 & 4 & $4(2,2)$ & $3(1,2)$ & 1234 & $\begin{array}{l}\text { B1IS', } \\
\text { B1SC, } \\
\text { B2IC, } \\
\text { B2SC }\end{array}$ \\
\hline Arrhenatherum elatius & $\mathrm{A}$ & & & & & & \\
\hline Aster linosyris & $\overline{\mathrm{A}}$ & & & & & & \\
\hline Asterolinum linum-stellatum & $\overline{A D}$ & B1B2 & 4 & $4(2,2)$ & $3(2,1)$ & 1234 & $\begin{array}{l}\text { B1SC, } \\
\text { B1SS', } \\
\text { B2IC, } \\
\text { B2SC }\end{array}$ \\
\hline Astragalus monspessulanus & $\overline{\mathrm{A}}$ & & & & & & \\
\hline
\end{tabular}


EVOLUCIÓN DE LA COMPOSICIÓN FLORÍSTICA POST-FUEGO EN UN CARRASCAL DE NAVARRA

\begin{tabular}{|c|c|c|c|c|c|c|c|}
\hline Taxa & Tiem. & Fueg. & Estr. & $\begin{array}{l}\text { Tot. } \\
\text { cuad. }\end{array}$ & $\begin{array}{l}\text { Final. } \\
\text { cuad. }\end{array}$ & Años & Cuadrados \\
\hline Avenula bromoides & AD & B1B2 & 4 & $2(1,1)$ & $2(1,1)$ & 234 & $\begin{array}{l}\text { B1IC, } \\
\text { B2SC }\end{array}$ \\
\hline Bellis perennis & $\mathrm{A}$ & & & & & & \\
\hline Bellis sylvestris & A & & & & & & \\
\hline Blackstonia perfoliata & $\mathrm{AD}$ & $\overline{\mathrm{B} 1}$ & 4 & 1 & 0 & 3 & B1SS $^{\prime}$ \\
\hline Bombycilaena erecta & $\mathrm{AD}$ & B2 & 4 & 1 & 0 & 3 & B2SC \\
\hline Brachypodium phoenicoides & $\mathrm{AD}$ & $\mathrm{B} 2$ & 2 & 1 & 0 & 12 & $\mathrm{~B}_{2} \mathrm{SS}^{\prime}$ \\
\hline Brachypodium retusum & AD & B1B2 & 2 & $8(4,4)$ & $8(4,4)$ & 1234 & $\begin{array}{l}\text { B1IC, } \\
\text { B1IS', } \\
\text { B1SC, } \\
\text { B1SS', } \\
\text { B2IC, } \\
\text { B2IS', } \\
\text { B2SC, } \\
\text { B2SS' }\end{array}$ \\
\hline Brimeura amethystina & A & & & & & & \\
\hline Bromus erectus & $\mathrm{AD}$ & B1 & 1 & 1 & 0 & 1 & B1IC \\
\hline Bupleurum baldense & A & & & & & & \\
\hline Bupleurum fruticescens & $\mathrm{A}$ & & & & & & \\
\hline Bupleurum rigidum & $\mathrm{A}$ & & & & & & \\
\hline Buxus sempervirens & $\mathrm{A}$ & & & & & & \\
\hline Campanula rotundifolia & $\mathrm{A}$ & & & & & & \\
\hline Carex hallerana & $\mathrm{AD}$ & B1 & 1 & 1 & 1 & 1234 & B1SS' $^{\prime}$ \\
\hline Carlina vulgaris & $\mathrm{A}$ & & & & & & \\
\hline Cerastium pumilum & $\mathrm{A}$ & & & 7 & & & \\
\hline Cerastium semidecandrum & D & $\mathrm{B} 2$ & 3 & 1 & 0 & 3 & B2SC \\
\hline Cirsium arvense & 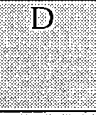 & $\mathrm{B} 1$ & 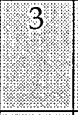 & 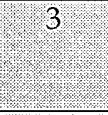 & 1.2. & 1234 & $\begin{array}{l}\mathrm{B}^{\prime \prime S} \\
\mathrm{~B}^{\prime} \\
\mathrm{B}^{\prime} \mathrm{SS}^{\prime}\end{array}$ \\
\hline Girsium vulgare & D & B1 B2 & 3. & $4(2,2)$ & $2(1,1)$ & 1234 & 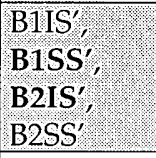 \\
\hline Cistus salvifolius & AD & B1B2 & 7 & $5(2,3)$ & $5(2,3)$ & 1234 & $\begin{array}{l}\text { B1IC, } \\
\text { B1IS', } \\
\text { B2IC, } \\
\text { B2IS', } \\
\text { B2SS' }^{\prime}\end{array}$ \\
\hline Clinopodium vulgare & $\mathrm{A}$ & & & & & & \\
\hline Conopodium majus & $\mathrm{AD}$ & B1B2 & 2 & $2(1,1)$ & $1(0,1)$ & 1234 & $\begin{array}{l}\text { B1SS', }^{\prime} \\
\text { B2SS }^{\prime}\end{array}$ \\
\hline Coronilla minima & $\mathrm{AD}$ & $\mathrm{B} 2$ & 4 & 1 & 0 & 12 & B2IS' \\
\hline
\end{tabular}


PIRINEOS 153-154

\begin{tabular}{|c|c|c|c|c|c|c|c|}
\hline Taxa & Tiem. & Fueg. & Estr. & $\begin{array}{l}\text { Tot. } \\
\text { cuad. }\end{array}$ & $\begin{array}{l}\text { Final. } \\
\text { cuad. }\end{array}$ & $A \tilde{n} O S$ & Cuadrados \\
\hline Crepis albida & A & & & & & & \\
\hline Crocus nevadensis & $\mathrm{AD}$ & B2 & 2 & 1 & 0 & 23 & B2SC \\
\hline Crocus nudiflorus & $\mathrm{AD}$ & B1B2 & 2 & $6(3,3)$ & 0 & 123 & $\begin{array}{l}\text { B1IS', } \\
\text { B1SC, } \\
\text { B1SS', } \\
\text { B2IC, } \\
\text { B2IS', } \\
\text { B2SC }\end{array}$ \\
\hline Crucianella angustifolia & AD & B1 & 4 & 1 & 1 & 34 & B1SC \\
\hline Dactylis glomerata & $\overline{A D}$ & B1 & 1 & 1 & 1 & 34 & B1IC \\
\hline $\begin{array}{l}\text { Daucus carota subsp. } \\
\text { maritimus }\end{array}$ & A & & & & & & $\therefore$ \\
\hline Daucus carota & A & & & & & & \\
\hline Desmazeria rigida & $\mathrm{A}$ & & & & & & \\
\hline Dianthus monspessulanum & $\mathrm{A}$ & & & & & & \\
\hline Dianthus hispanicus & $\mathrm{AD}$ & B2 & 1 & 1 & 1 & 234 & B2SC \\
\hline Dorycnium pentaphyllum & $\mathrm{AD}$ & $\mathrm{B} 1 \mathrm{~B} 2$ & 7 & $5(2,3)$ & $4(2,2)$ & 1234 & B1IS', \\
\hline & & & & & & & $\begin{array}{l}\text { B1SS', } \\
\text { B2IC, } \\
\text { B2IS', } \\
\text { B2SS' }^{\prime}\end{array}$ \\
\hline Erica vagans & $\overline{A D}$ & B1B2 & 5 & $3(2,1)$ & $3(2,1)$ & 1234 & $\begin{array}{l}\text { B1IC, } \\
\text { B1IS', } \\
\text { B2IS' }\end{array}$ \\
\hline Eryngium campestre & AD & B1 & 4 & 1 & 1 & 24 & B1IC \\
\hline Euphorbia exigua & AD & B1B2 & 4 & $2(1,1)$ & $2(1,1)$ & 234 & $\begin{array}{l}\text { B1SS', } \\
\text { B2SC }\end{array}$ \\
\hline Euphorbia minuta & $\mathrm{AD}$ & B2 & 1 & 1 & 1 & 1234 & B2IS' $^{\prime}$ \\
\hline Festuca grupo rubra & $\mathrm{AD}$ & $\mathrm{B} 1 \mathrm{~B} 2$ & 1 & $7(4,3)$ & $1(2,0)$ & 1234 & $\begin{array}{l}\text { B1IC, } \\
\text { B1IS', } \\
\text { B1SC, } \\
\text { B1SS' } \\
\text { B2IC, } \\
\text { B2IS', }^{\prime} \text { B2SS' }\end{array}$ \\
\hline Filipendula vulgaris & $\overline{A D}$ & B1B2 & 2 & $3(2,1)$ & $2(1,1)$ & 1234 & $\begin{array}{l}\text { B1IC, } \\
\text { B1SS', } \\
\text { B2SS' }\end{array}$ \\
\hline Fritillaria pyrenaica & $\mathrm{AD}$ & B1B2 & 2 & $2(1,1)$ & 0 & 12 & $\begin{array}{l}\text { B1SS', } \\
\text { B2SS' }^{\prime}\end{array}$ \\
\hline Fumana ericoides & $\mathrm{AD}$ & B2 & 4 & 1 & 1 & 1234 & B2SC \\
\hline Galium aparine & $\mathrm{D}$ & $\mathrm{B} 2$ & 3 & 1 & 0 & 3 & $\mathrm{~B} 2 \mathrm{SS}$ \\
\hline
\end{tabular}


EVOLUCIÓN DE LA COMPOSICIÓN FLORÍSTICA POST-FUEGO EN UN CARRASCAL DE NAVARRA

\begin{tabular}{|c|c|c|c|c|c|c|c|}
\hline Taxa & Tiem. & Fueg. & Estr. & $\begin{array}{l}\text { Tot. } \\
\text { cuad. }\end{array}$ & $\begin{array}{l}\text { Final. } \\
\text { cuad. }\end{array}$ & $A \tilde{n} o s$ & Cuadrados \\
\hline Galium fruticescens & $\mathrm{AD}$ & $\mathrm{B} 2$ & 1 & 1 & 1 & 1234 & B2IC \\
\hline Galium sp. & $\mathrm{AD}$ & B2 & 1 & 2 & 1 & 1234 & $\begin{array}{l}\mathrm{B} 2 \mathrm{IS}^{\prime}, \\
{\mathrm{B} 2 S S^{\prime}}\end{array}$ \\
\hline Gastridium ventricosum & $\mathrm{AD}$ & B1B2 & 4 & $2(1,1)$ & $2(1,1)$ & 234 & $\begin{array}{l}\text { B1SC, } \\
\text { B2SC }\end{array}$ \\
\hline $\begin{array}{l}\text { Genista hispanica subsp. } \\
\text { occidentalis }\end{array}$ & $\mathbf{A D}$ & B1B2 & 7 & $6(3,3)$ & $6(3,3)$ & 12.34 & $\begin{array}{l}\text { B1IC, } \\
\text { B1IS', } \\
\text { B1SC, } \\
\text { B2IC, } \\
\text { B2IS', } \\
\text { B2SS' }\end{array}$ \\
\hline Genista scorpius & AD & B2 & 7 & 2 & 2 & 1234 & $\begin{array}{l}\text { B2IC, } \\
\text { B2IS' }\end{array}$ \\
\hline Geum sylvaticum & $\mathrm{A}$ & & & & & & \\
\hline Gladiolus illyricus & $\mathrm{A}$ & & & & & & \\
\hline Globularia vulgaris & A & & & & & & \\
\hline Helianthemum apenninum & $\mathrm{A}$ & . & & & & & \\
\hline Helianthemum canum & $\mathrm{AD}$ & $\mathrm{B} 1 \mathrm{~B} 2$ & 5 & $2(1,1)$ & $1(0,1)$ & 234 & $\begin{array}{l}\text { B1IS', } \\
\text { B2SC }\end{array}$ \\
\hline Hordeum sp. & D & B2 & 3 & 1 & 0 & 2 & $\mathrm{~B} 2 \mathrm{SS}$ \\
\hline Inula montana & $\mathrm{A}$ & & & & & & \\
\hline Juniperus communis & $\mathrm{A}$ & & & & & & \\
\hline Juniperus oxycedrus & A & & & & & & \\
\hline Koeleria vallesiana & $\mathrm{A}$ & & & & & & \\
\hline Lavandula latifolia & $\mathrm{A}$ & & & & & & \\
\hline Leuzea conifera & $\mathrm{A}$ & & & & & & \\
\hline Linum narbonense & $\mathrm{A}$ & & & & & & \\
\hline Lonicera implexa & $\mathrm{A}$ & & & & & & \\
\hline Merendera pyrenaica & A & & & & & & \\
\hline Muscari neglectum & $\mathrm{AD}$ & B2 & 2 & 2 & 0 & 2 & $\begin{array}{l}\text { B2IC, } \\
\text { B2SC }\end{array}$ \\
\hline Narcissus assoanus & AD & B1B2 & 2 & $4(1,3)$ & 0 & 123 & $\begin{array}{l}\text { B1IS', } \\
\text { B2IC, } \\
\text { B2SC, } \\
\text { B2SS' }\end{array}$ \\
\hline Narcissus asturiensis & $\mathrm{A}$ & & & & & & \\
\hline Ononis minutissima & $\mathrm{AD}$ & B1B2 & 4 & $2(1,1)$ & $1(0,1)$ & 1234 & $\begin{array}{l}\text { B1SS', } \\
\text { B2SC }\end{array}$ \\
\hline Ophrys fusca & $\mathrm{A}$ & & & & & & . \\
\hline $\begin{array}{l}\text { Orchis morio subsp. } \\
\text { champagneuxii }\end{array}$ & $\mathrm{A}$ & & & & & & \\
\hline Orchis provincialis & $\mathrm{A}$ & & & & & & \\
\hline
\end{tabular}


PIRINEOS 153-154

\begin{tabular}{|c|c|c|c|c|c|c|c|}
\hline Taxa & Tiem. & Fueg. & Estr. & $\begin{array}{c}\text { Tot. } \\
\text { culad. }\end{array}$ & $\begin{array}{l}\text { Final. } \\
\text { cuad. }\end{array}$ & Años & Cuadrados \\
\hline Orchis ustulata & A & & & & & & \\
\hline Phleum pratense & $\mathrm{A}$ & & & & & & \\
\hline Phlomis lychnitis & $\overline{A D}$ & B2 & 1 & 1 & 0 & 12 & B2IC \\
\hline Picris echioides & D. & $\mathrm{B} 2$ & 3 & 1 & 0 & 1 . & B2IC \\
\hline Picris hieracioides & $\mathrm{B}$ & $\mathrm{B} 2 \mathrm{Y}$ & 3 & 11 & 0 & 2 & B2IC \\
\hline Polygala vulgaris & AD & B2 & 1 & 1 & 0 & 23 & B2IS $^{\prime}$ \\
\hline Potentilla montana & A & & & & & & \\
\hline Potentilla reptans & D & B2 & 3 & 1 & 1 & 234 & B2IS $^{\prime}$ \\
\hline Potentilla tabernaemontani & $\mathrm{AD}$ & B1B2 & 1 & $2(1,1)$ & $1(1,0)$ & 1234 & $\begin{array}{l}\text { B1SS', } \\
\text { B2SS' }\end{array}$ \\
\hline Prunella laciniata & A & & & & & & \\
\hline Prunus spinosa & $\mathrm{A}$ & & & & & & \\
\hline Quercus coccifera & $\mathrm{A}$ & & & & & & \\
\hline Quercus faginea & $\overline{A D}$ & B2 & 1 & 1 & 1 & 1234 & B2IS' $^{\prime}$ \\
\hline Quercus rotundifolia & AD & B1B2 & 1 & $8(4,4)$ & $8(4,4)$ & 1234 & $\begin{array}{l}\text { B1IC, } \\
\text { B1IS', } \\
\text { B1SC, } \\
\text { B1SS', } \\
\text { B2IC, } \\
\text { B2IS'; } \\
\text { B2SC, } \\
\text { B2SS' }\end{array}$ \\
\hline Ranunculus gramineus & A & & & & & & \\
\hline Rhamnus alaternus & AD & B1 & 5 & 1 & 1 & 134 & B1SS $^{\prime}$ \\
\hline Rhamnus saxatilis & $\mathrm{AD}$ & B1 & 1 & 1 & 0 & 1 & ${\mathrm{~B} 1 S S^{\prime}}^{\prime}$ \\
\hline Rubia peregrina & $\overline{A D}$ & B1B2 & 2 & $5(4,1)$ & $5(4,1)$ & 1234 & $\begin{array}{l}\text { B1IC, } \\
\text { B1IS', } \\
\text { B1SC, } \\
\text { B1SS', } \\
\text { B2IS' }\end{array}$ \\
\hline Ruscus aculeatus & A & & & & & & \\
\hline Santolina chamaecyparissus & A & & & & & & \\
\hline Scorzonera graminifolia & $\mathrm{AD}$ & B1B2 & 1 & $3(1,2)$ & 0 & 123 & $\begin{array}{l}\text { B1IC, } \\
\text { B2IS', } \\
\text { B2SC }\end{array}$ \\
\hline Scorzonera hispanica & $\mathrm{A}$ & & & & & & \\
\hline Sedum sediforme & A & & & & & & \\
\hline Silene nutans & $\overline{\mathrm{AD}}$ & $\overline{\mathrm{B} 2}$ & 5 & 1 & 0 & 1 & B2IS' \\
\hline Sonchus asper & $\mathrm{D}$ & B2 & 3 & 1 & 0 & 1 & B2IC \\
\hline Sonchus oleracents & 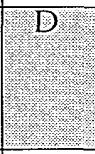 & $\mathrm{B} 1 \mathrm{~B} 2$ & 2.0 .9 & $4(2,2)$ & 0 & & $\begin{array}{l}\text { B11C, } \\
\text { B1SS' } \\
\text { B2IS' } \\
\text { B2SS' }\end{array}$ \\
\hline
\end{tabular}


EVOLUCIÓN DE LA COMPOSICIÓN FLORÍSTICA POST-FUEGO EN UN CARRASCAL DE NAVARRA

\begin{tabular}{|c|c|c|c|c|c|c|c|}
\hline Taxa & Tiem. & Fueg. & $\overline{\text { Estr. }}$ & $\begin{array}{l}\text { Tot. } \\
\text { cuad. }\end{array}$ & $\begin{array}{l}\text { Final. } \\
\text { cuad. }\end{array}$ & Años & Cuadrados \\
\hline Stachys officinalis & $\mathrm{A}$ & & & & & & \\
\hline Tanacetum corymbosum & $\mathrm{A}$ & & & & & & \\
\hline Taraxacum sp. & $\mathrm{D}$ & B1 & 3 & 1 & 0 & 3 & B1IC \\
\hline Teucrium chamaedrys & AD & B1B2 & 1 & $4(1,3)$ & $4(1,3)$ & 1234 & $\begin{array}{l}\text { B1IC, } \\
\text { B2IS', } \\
\text { B2SC, } \\
\text { B2SS' }^{\prime}\end{array}$ \\
\hline Teucrium pyrenaicum & $\mathrm{A}$ & & & & & & \\
\hline $\begin{array}{l}\text { Teucrium polium subsp. } \\
\text { capitatum }\end{array}$ & A & & & & & & \\
\hline Thalictrum tuberosum & $\mathrm{AD}$ & B1B2 & 2 & $5(2,3)$ & $3(2,1)$ & 1234 & $\begin{array}{l}\text { B1IC, } \\
\text { B1IS', } \\
\text { B2IC, } \\
\text { B2SC, } \\
\text { B2SS' }\end{array}$ \\
\hline Thesium divaricatum & $\mathrm{AD}$ & B2 & 8 & 1 & 1 & 1234 & B2IS' $^{\prime}$ \\
\hline Thymelaea ruizii & $\mathrm{AD}$ & B1B2 & 1 & $2(1,1)$ & $2(1,1)$ & 1234 & $\begin{array}{l}\text { B1SC, } \\
\text { B2IS' }\end{array}$ \\
\hline Thymus vulgaris & $\overline{A D}$ & B1B2 & 5 & $3(1,2)$ & $3(1,2)$ & 1234 & $\begin{array}{l}\text { B1SC, } \\
\text { B2IS', } \\
\text { B2SC }\end{array}$ \\
\hline Trinia glauca & $\mathrm{A}$ & & & & & & \\
\hline Vicia-Lathyrus & D & B2 & 3 & 1 & 1 & 24 & B2IS' $^{\prime}$ \\
\hline Viola alba & $\mathrm{A}$ & & & & & & \\
\hline Viola hirta & $\mathrm{AD}$ & B1B2 & 5 & $4(2,2)$ & $3(1,2)$ & 1234 & $\begin{array}{l}\text { B1IC, } \\
\text { B1SS', } \\
\text { B2SC, } \\
\text { B2SS' }^{\prime}\end{array}$ \\
\hline Compuesta 1 & & B2 & $3-4$ & 1 & 0 & 2 & $\mathrm{~B} 2 \mathrm{IS}^{\prime}$ \\
\hline Cotiledones 1 & & B1 & $3-4$ & 1 & 0 & 2 & B1IS' \\
\hline Cotiledones 2 & & B1 & $3-4$ & 1 & 0 & 1 & B1SS' $^{\prime}$ \\
\hline Cotiledones 3 & & B1 & $3-4$ & 1 & 0 & 1 & B1SS' $^{\prime}$ \\
\hline Cotiledones 4 & & B1B2 & $3-4$ & $4(1,3)$ & 0 & 12 & B1SS', \\
\hline & & & & & & & $\begin{array}{l}\text { B2IC, } \\
\text { B2SC, } \\
\text { B2SS' }^{\prime}\end{array}$ \\
\hline Cotiledones 5 & & B2 & $3-4$ & 1 & 0 & 2 & B2SC \\
\hline Crucífera 1 & & B2 & $3-4$ & 1 & 0 & 2 & B2IS' $^{\prime}$ \\
\hline Poacea 1 & & B1 & - & 1 & 0 & 2 & B1IC \\
\hline Poacea 2 & & B1 & - & 1 & 0 & 2 & B1IS' $^{\prime}$ \\
\hline Poacea 3 & & B1B2 & - & $2(1,1)$ & 0 & 1 & $\begin{array}{l}\text { B1SS' }^{\prime} \\
\text { B2SS }^{\prime}\end{array}$ \\
\hline
\end{tabular}




\begin{tabular}{|c|c|c|c|c|c|c|c|}
\hline Taxa & Tiem. & Fueg. & Estr. & $\begin{array}{l}\text { Tot. } \\
\text { cuad. }\end{array}$ & $\begin{array}{l}\text { Final. } \\
\text { cuad. }\end{array}$ & Años & Cuadrados \\
\hline Poacea 4 & & B1 & - & 1 & 0 & 2 & B1SS' \\
\hline Poacea 5 & & B2 & - & 1 & 0 & 2 & B2SC \\
\hline TOTALES & $\begin{array}{l}\text { A: } 58 \\
\text { D: } 12 \\
\text { AD: } 56 \\
\text { ?: } 12\end{array}$ & $\begin{array}{l}\text { B1: } 16 \\
\text { B2: } 31 \\
\text { B1B2:33 }\end{array}$ & $\begin{array}{l}\text { Rebr: } 30 \\
\text { Germ:33 } \\
\text { R-ger:12 } \\
?: 5\end{array}$ & & & & \\
\hline
\end{tabular}

Tabla 4. Tiempo de aparición de los taxa: A, sólo antes de los fuegos; D, sólo después; AD, antes y después. Tipo de fuego en el que han aparecido tras los incendios: B1, sólo fuego rápido; B2, sólo fuego lento; B1B2, ambos tipos de fuego. Estrategias de regeneración: 1, plantas rebrotadoras mediante órganos superficiales; 2 , plantas rebrotadoras mediante órganos subterráneos; 3 , plantas germinadoras oportunistas; 4 , plantas germinadoras no oportunistas; 5 , plantas rebrotadoras mediante órganos superficiales y germinadoras; 6 , plantas rebrotadoras mediante órganos subterráneos y germinadoras; 7 , plantas rebrotadoras mediante órganos superficiales y germinadoras, preferentemente germinadoras; 8 , plantas rebrotadoras mediante órganos superficiales y germinadoras, preferentemente rebrotadoras; -, estrategia desconocida. Time of appearance of the taxa: $A$, only before the fires; $D$, only after; $A D$, before and after. Type of fire in which they have appeared after the fires: B1, only canopy fire, B2, only ground fire; B1B2, both fire types. Regeneration strategies: 1, resprouting plants by superficial organs; 2, resprouting plants by subterranean organs; 3 , germinating opportunist plants; 4 , germinating not opportunist plants; 5 , resprouting plants by superficial organs and germinating plants; 6 , resprouting plants by subterranean organs and germinating plants; 7, resprouting plants by superficial organs and germinating plants, preferably the last ones; 8 , resprouting plants by superficial organs and germinating plants, preferably resprouting plants; -, unknown strategy. 